Supporting Information for:

\title{
anti-Selective Asymmetric Henry Reaction Catalyzed by a Heterobimetallic Cu-Sm-Aminophenol Sulfonamide Complex
}

\author{
Yang Li, ${ }^{\dagger}$ Ping Deng, ${ }^{\dagger}$ Youmao Zeng, ${ }^{\dagger}$ Yan Xiong, ${ }^{\ddagger}$ Hui Zhou ${ }^{*}{ }^{\dagger}$ \\ hzhou@cqmu.edu.cn \\ 'School of Pharmaceutical Science, Chongqing Medical University, Chongqing, China \\ ${ }^{\star}$ School of Chemistry and Chemical Engineering, Chongqing University, Chongqing, China
}

\section{Contents:}

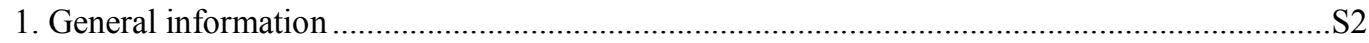

2. General procedures for the preparation of the chiral ligand......................................................S2

3. General Procedure for Catalytic Asymmetric Henry Reaction ................................................S3

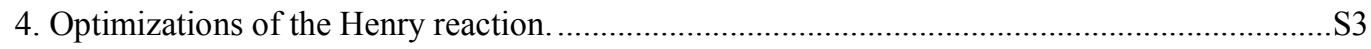

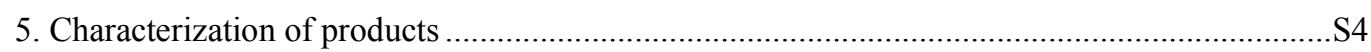

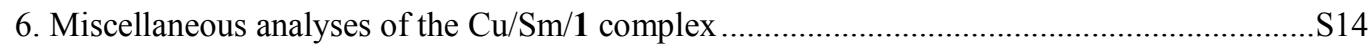

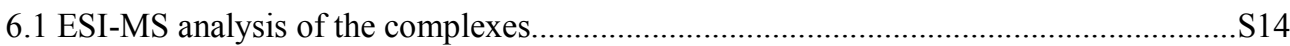

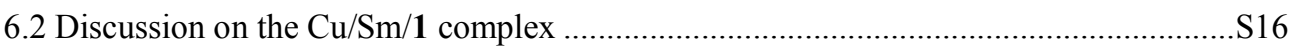

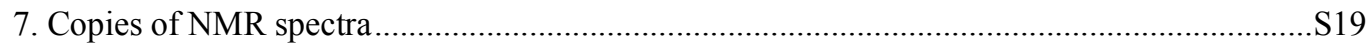




\section{General information}

All reagents were obtained from Adamas, Aladin, or Acros etc. without further purification unless otherwise noted. High resolution mass spectra were measured on commercial instruments. NMR spectra were recorded on commercial instruments, and operating at $500 \mathrm{MHz}$ for ${ }^{1} \mathrm{HNMR}$ and $125 \mathrm{MHz}$ for ${ }^{13} \mathrm{C}$ NMR. Chemical shifts were reported in ppm from tetramethylsilane with the solvent resonance as the internal standard $\left(\mathrm{CDCl}_{3}, \delta=7.26\right)$ in ${ }^{1} \mathrm{H}$ NMR spectra and Chemical shifts were reported in ppm from the tetramethylsilane with the solvent resonance as internal standard $\left(\mathrm{CDCl}_{3}, \delta=77.0\right)$ in ${ }^{13} \mathrm{C}$ NMR spectra. Spectra are reported as follows: chemical shift $(\delta$ ppm), multiplicity ( $\mathrm{s}=$ singlet, $\mathrm{d}=$ doublet, $\mathrm{t}=$ triplet, $\mathrm{q}=$ quartet, $\mathrm{m}=$ multiplet), coupling constants $(\mathrm{Hz})$, integration, and assignment. The enantiomeric excess (ee) was determined by HPLC analysis. Analytical HPLC was performed on a Shimadzu liquid chromatography equipped and agilent 1260, using a chiral DAICEL CHIRALCEL OD-H or CHIRALPAK AD-H or CHIRALPAK AS-H column at $210 \mathrm{~nm}$. The ratio of anti/syn was determined by ${ }^{1} \mathrm{H}$ NMR spectroscopy or HPLC analysis.

\section{General procedures for the preparation of the chiral ligand.}

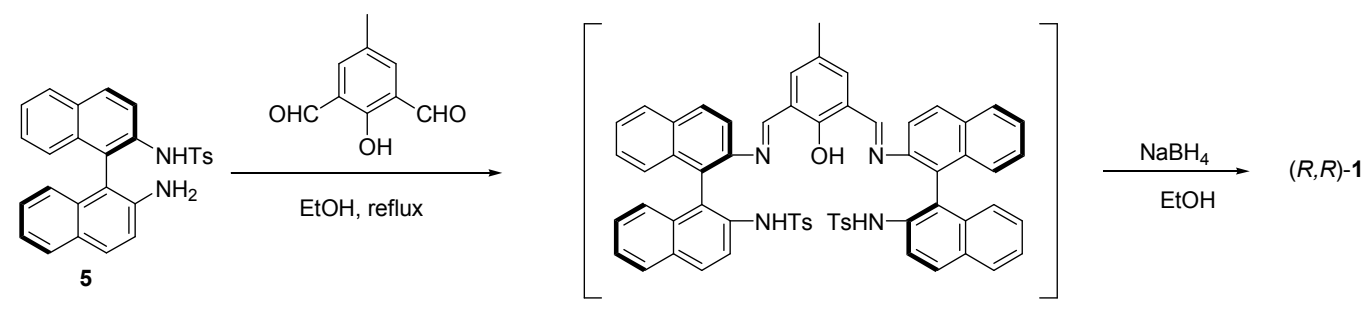

To a solution of $5(2 \mathrm{mmol})$ in $50 \mathrm{~mL}$ ethanol was slowly added the dialdehyde $(1 \mathrm{mmol})$, and the mixture was stirred at $85{ }^{\circ} \mathrm{C}$ until the end of the reaction detected by TLC. Sodium borohydride $(8 \mathrm{mmol})$ was then added in small portions at $0{ }^{\circ} \mathrm{C}$. And then the reaction was allowed to warm to ambient temperature for $12 \mathrm{~h}$. It was poured over $100 \mathrm{~mL}$ of water and extracted with $\mathrm{CH}_{2} \mathrm{Cl}_{2}$. The combined organic phase was washed with Sat. $\mathrm{NaCl}$ and dried with $\mathrm{Na}_{2} \mathrm{SO}_{4}$, and the solvents were removed in vacuo and purified through column chromatography (petroleum ether / AcOEt, 7:1) on silica gel to give $(R, R)-\mathbf{1}$ as a flaxen solid, $35 \%$ yield, $0.35 \mathrm{~g}$.

${ }^{1} \mathrm{H}$ NMR (500 MHz, $\left.\mathrm{CDCl}_{3}\right): \delta$ 8.08-8.05 (m, 2H), 7.91-7.88 (m, 2H), 7.86-7.83 (m, 2H), 7.79-7.76 (m, 4H), 7.41-7.38 (m, 4H), 7.35-7.30 (m, 2H), 7.23-7.16 (m, 4H), 7.04-7.02 (m, 4H), 7.00- $6.97(\mathrm{~m}, 2 \mathrm{H}), 6.95-6.91(\mathrm{~m}, 2 \mathrm{H}), 6.69(\mathrm{~s}, 2 \mathrm{H}), 6.57(\mathrm{~s}, 2 \mathrm{H}), 6.39-6.37(\mathrm{~m}, 2 \mathrm{H}), 4.24-4.15(\mathrm{~m}$, 4H), 2.31 (s, 6H), 2.13 (s, 3H). ${ }^{13} \mathrm{C}$ NMR (125 MHz, $\left.\mathrm{CDCl}_{3}\right): \delta$ 152.1, 143.7, 136.1, 133.9, 133.0, $132.8,131.1,130.8,129.8,129.4,128.1,128.0,127.7,127.2,127.0,125.2,124.0,123.2,122.6$, 
120.1, 118.7, 115.0, 45.8, 21.4, 20.3. HRMS (ESI): $\mathrm{m} / z$ calcd for $\mathrm{C}_{63} \mathrm{H}_{53} \mathrm{~N}_{4} \mathrm{O}_{5} \mathrm{~S}_{2}[\mathrm{M}+\mathrm{H}]^{+}$: 1009.34519, found 1009.34282.

\section{General Procedure for Catalytic Asymmetric Henry Reaction}

Method A (one-pot): The mixture of $\mathrm{Cu}(\mathrm{OAc})_{2}(0.02 \mathrm{mmol}), \mathrm{Sm}(\mathrm{O} i \mathrm{Pr})_{3}(0.02 \mathrm{mmol})$, and $(R, R)-1(0.02 \mathrm{mmol})$ was stirred in THF $(0.5 \mathrm{~mL})$ at $35^{\circ} \mathrm{C}$ for $2 \mathrm{~h}$. The mixture was cooled to -30 ${ }^{\circ} \mathrm{C}$ followed by the addition of the aldehyde $(0.2 \mathrm{mmol})$, then nitroethane $(0.2 \mathrm{ml}), i \operatorname{Pr}_{2} \mathrm{NEt}$ (diluted in 0.2 $\mathrm{ml}$ THF, $30-50 \mathrm{~mol} \%$ ) and $0.2 \mathrm{ml}$ THF were added to the mixture. The stirring was continued for indicated time in Table 2 at $-30{ }^{\circ} \mathrm{C}$. The residue was purified by silica gel flash column chromatography (petroleum ether / AcOEt, 8:1 to 15:1, or petroleum ether / ether, 3:1) to afford the products.

Method B [stepwise, M1 $=\mathbf{C u}(\mathrm{OAc})_{2}, \mathrm{M} 2=\operatorname{Sm}(\mathrm{O}-i \mathrm{Pr})_{3}$ ]: The mixture of $\mathrm{Cu}(\mathrm{OAc})_{2}$ $(0.02 \mathrm{mmol})$ and $(R, R)-1(0.02 \mathrm{mmol})$ was stirred in THF $(0.3 \mathrm{~mL})$ at $35{ }^{\circ} \mathrm{C}$ for $1 \mathrm{~h}$. Then the mixture was transferred to another tube which was charged with $\mathrm{Sm}(\mathrm{OiPr})_{3}(0.02 \mathrm{mmol})$ with the help of THF $(0.4 \mathrm{~mL})$. $i \mathrm{Pr}_{2} \mathrm{NEt}$ (diluted in $0.2 \mathrm{ml} \mathrm{THF}, 30 \mathrm{~mol} \%$ ) was added to the mixture and the mixture containing $\mathrm{Cu}$ and $\mathrm{Sm}$ was stirred at $35^{\circ} \mathrm{C}$ for another $1 \mathrm{~h}$. The mixture was cooled to $-30{ }^{\circ} \mathrm{C}$ followed by the addition of the aldehyde $(0.2 \mathrm{mmol})$ and nitroethane $(0.2 \mathrm{ml})$. The stirring was continued for $90 \mathrm{~h}$ at $-30{ }^{\circ} \mathrm{C}$. The residue was purified by silica gel flash column chromatography (petroleum ether / AcOEt, 8:1) to afford the products.

Method C [stepwise, M1 $\left.=\mathbf{S m}(\mathbf{O}-i \mathrm{Pr})_{3}, \mathbf{M 2}=\mathbf{C u}(\mathbf{O A c})_{2}\right]$ : The mixture of $\mathrm{Sm}(\mathrm{O} i \mathrm{Pr})_{3}$ $(0.02 \mathrm{mmol})$ and $(R, R)-1(0.02 \mathrm{mmol})$ was stirred in THF $(0.3 \mathrm{~mL})$ at $35{ }^{\circ} \mathrm{C}$ for $1 \mathrm{~h}$. Then the mixture was transferred to another tube which was charged with $\mathrm{Cu}(\mathrm{OAc})_{2}(0.02 \mathrm{mmol})$ with the help of THF (0.4 mL). $i \operatorname{Pr}_{2} \mathrm{NEt}$ (diluted in $0.2 \mathrm{ml} \mathrm{THF}, 30 \mathrm{~mol} \%$ ) was added to the mixture and the mixture containing $\mathrm{Cu}$ and $\mathrm{Sm}$ was stirred at $35^{\circ} \mathrm{C}$ for another $1 \mathrm{~h}$. The mixture was cooled to $-30{ }^{\circ} \mathrm{C}$ followed by the addition of the aldehyde $(0.2 \mathrm{mmol})$ and nitroethane $(0.2 \mathrm{ml})$. The stirring was continued for $90 \mathrm{~h}$ at $-30{ }^{\circ} \mathrm{C}$. The residue was purified by silica gel flash column chromatography (petroleum ether / AcOEt, 8:1) to afford the products.

\section{Optimizations of the Henry reaction.}

The selected optimizations of the reaction are shown in Table S1. As the low reactivity, several tert-amine were examined and $i \mathrm{Pr}_{2} \mathrm{NEt}$ gave the best results (Entry 4 vs entries 1-3, Table $\mathrm{S} 1$ ). The increasing amount of $i \mathrm{Pr}_{2} \mathrm{NEt}$ and nitroethane could help to achieve better results (Entries 4-6 and entries 6-8, Table S1). When the reaction temperature was lowered to $-30{ }^{\circ} \mathrm{C}$, the 
diastereoselectivity and the enantioselectivity were both increased with the decreased reactivity, while increasing the amount of the base could improve the reactivity (Entries 8-10, Table S1).

Table S1. Selected optimizations of the Henry reaction. ${ }^{a}$

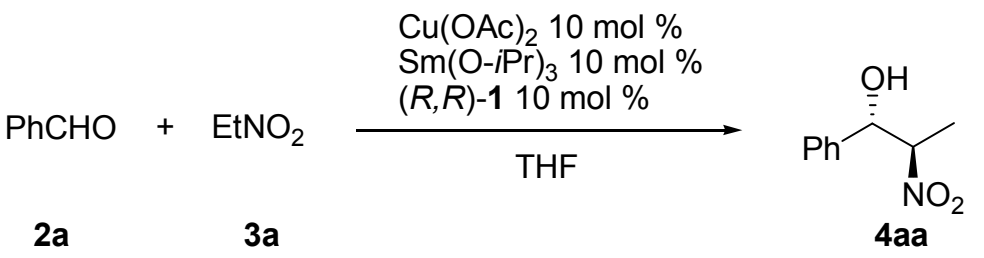

\begin{tabular}{|c|c|c|c|c|c|c|c|}
\hline entry & base & $\begin{array}{c}t \\
(\mathrm{~h})\end{array}$ & $\begin{array}{l}\text { temp } \\
\left({ }^{\circ} \mathrm{C}\right)\end{array}$ & $\begin{array}{l}\text { the amount of } \\
\text { the base } \\
(\mathrm{mol} \%)\end{array}$ & $\begin{array}{l}\text { yield } \\
(\%)^{b}\end{array}$ & $\begin{array}{c}\text { anti/syn } \\
(\%)^{c}\end{array}$ & $\begin{array}{c}\text { ee }(\%)^{c} \\
(\text { anti) }\end{array}$ \\
\hline 1 & none & 95 & 0 & - & trace & $4: 1$ & 72 \\
\hline 2 & $\mathrm{~N}$-methylmorpholine & 96 & 0 & 10 & 41 & $7: 1$ & 80 \\
\hline 3 & $\mathrm{Et}_{3} \mathrm{~N}$ & 43 & 0 & 10 & 98 & $6: 1$ & 84 \\
\hline 4 & $i \mathrm{Pr}_{2} \mathrm{NEt}$ & 43 & 0 & 10 & 74 & $8: 1$ & 84 \\
\hline 5 & $i \operatorname{Pr}_{2} \mathrm{NEt}$ & 43 & 0 & 5 & 49 & $8: 1$ & 85 \\
\hline 6 & $i \mathrm{Pr}_{2} \mathrm{NEt}$ & 43 & 0 & 20 & 81 & $7: 1$ & 86 \\
\hline $7^{d}$ & $i \mathrm{Pr}_{2} \mathrm{NEt}$ & 43 & 0 & 20 & 30 & $7: 1$ & 84 \\
\hline $8^{e}$ & $i \mathrm{Pr}_{2} \mathrm{NEt}$ & 43 & 0 & 20 & 94 & $8: 1$ & 86 \\
\hline $9^{e}$ & $i \mathrm{Pr}_{2} \mathrm{NEt}$ & 90 & -30 & 20 & 75 & $12: 1$ & 93 \\
\hline $10^{e}$ & $i \operatorname{Pr}_{2} \mathrm{NEt}$ & 90 & -30 & 30 & 90 & $14: 1$ & 93 \\
\hline \multicolumn{8}{|c|}{$\begin{array}{l}{ }^{a} \text { Unless otherwise noted all reactions were carried out on a } 0.2 \mathrm{mmol} \text { scale (benzaldehyde) with nitroethane }(0.1 \\
\mathrm{mL}) \text { in THF }(0.9 \mathrm{~mL}) \text { in the presence of }(R, R)-1(10 \mathrm{~mol} \%), \mathrm{Cu}(\mathrm{OAc})_{2}(10 \mathrm{~mol} \%) \text { and } \mathrm{Sm}(\mathrm{OiPr})_{3}(10 \mathrm{~mol} \%) \text { and } \\
\text { the base. The catalyst was prepared in accordance with Method B. }{ }^{b} \text { Isolated yield. }{ }^{c} \text { Determined by chiral HPLC } \\
\text { analysis (Chiralpak AD-H). }{ }^{d} 50 \mu \mathrm{l} \text { nitroethane was used. }{ }^{e} 0.2 \mathrm{~mL} \text { nitroethane was used. }\end{array}$} \\
\hline
\end{tabular}

\section{Characterization of products}

The relative and absolute configurations of anti-products were assigned by comparison with ${ }^{1} \mathrm{H}$ NMR and HPLC data in literature. ${ }^{[1]}$ The absolute configuration of the 4ca, 4ia, 4oa was determined by analogy.

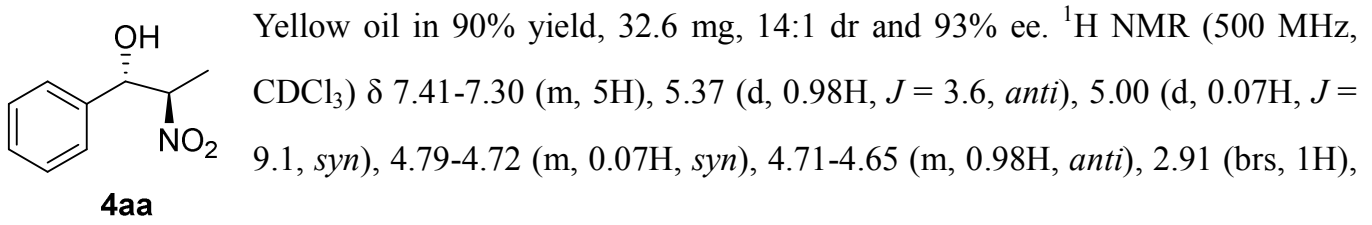

[1]. (a) Ooi, T.; Uraguchi, D.; Sakaki, S. J. Am. Chem. Soc. 2007, 129, 12392-12393. (b) Mei, H.; Xiao, X.; Lin, L. Feng, X. J. Org. Chem. 2015, 80, 2272-2280. (c) Handa, S.; Nagawa, K.; Sohtome, Y.; Matsunaga, S.; Shibasaki, M. Angew. Chem. Int. Ed. 2008, 47, 3230-3233. (d) Qin, H.; Yu, W.; Zhou, J.; Zhang, Y.; Ruan, Y.; Zhou, Z.; Chen, H. Chem. Eur. J. 2013, 19, 16541-16544. 
$1.48(\mathrm{~d}, 3 \mathrm{H}, J=6.8)$. HPLC (CHIRALCEL AD-H column, hexane/2-propanol = 90/10, flow 0.8 $\mathrm{mL} / \mathrm{min}$, detection at $210 \mathrm{~nm}):(1 S, 2 R) \mathrm{t}_{\mathrm{R}}($ anti major $)=9.7 \mathrm{~min},(1 R, 2 S) \mathrm{t}_{\mathrm{R}}($ anti minor $)=10.4$ $\min ,(1 S, 2 S) \mathrm{t}_{\mathrm{R}}(s y n)=12.0 \mathrm{~min},(1 R, 2 R) \mathrm{t}_{\mathrm{R}}($ syn $)=13.2 \mathrm{~min}$.
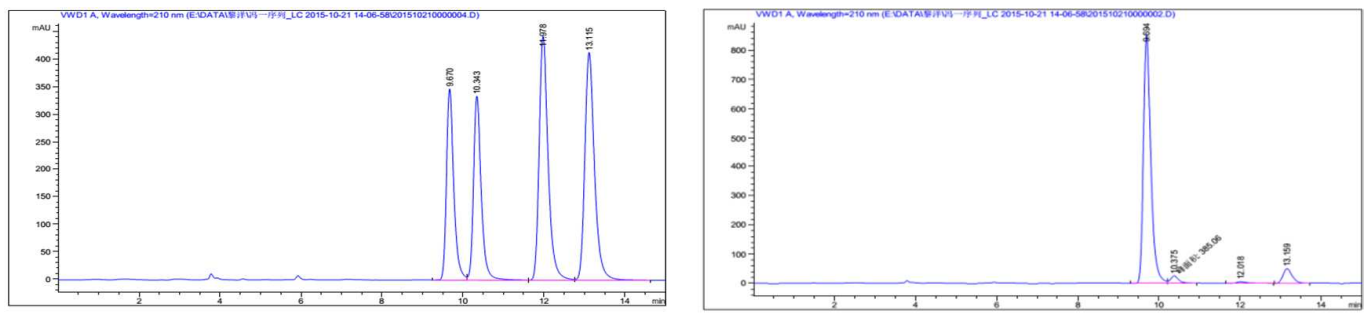

\begin{tabular}{llll}
\hline Peak\# & Retention Time & Area & Area $\%$ \\
\hline 1 & 9.7 & 10787 & 96.553 \\
2 & 10.4 & 385 & 3.447 \\
Total & & 11172 & 100.000 \\
\hline
\end{tabular}

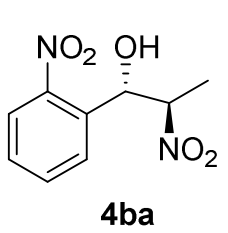

Yellow oil in 99\% yield, $45.0 \mathrm{mg},>30: 1 \mathrm{dr}$ and $96 \%$ ee. ${ }^{1} \mathrm{H}$ NMR $(500 \mathrm{MHz}$, $\left.\mathrm{CDCl}_{3}\right) \delta 8.01(\mathrm{dd}, 1 \mathrm{H}, J=1.1), 7.87-7.85(\mathrm{~m}, 1 \mathrm{H}), 7.68-7.64(\mathrm{~m}, 1 \mathrm{H})$, $7.48-7.44(\mathrm{~m}, 1 \mathrm{H}), 6.02(\mathrm{~d}, 0.97 \mathrm{H}, J=2.3$, anti), $5.64(\mathrm{~d}, 0.03 \mathrm{H}, J=6.6$, syn), 4.94-4.88 (m, 1H, anti+syn), 3.27 (brs, $1 \mathrm{H}), 1.44(\mathrm{~d}, 3 \mathrm{H}, J=7.0)$. HPLC

(CHIRALCEL OD-H+AD-H column, hexane $/ 2$-propanol $=90 / 10$, flow $0.5 \mathrm{~mL} / \mathrm{min}$, detection at $210 \mathrm{~nm}): \mathrm{t}_{\mathrm{R}}($ anti minor $)=50.5 \mathrm{~min}, \mathrm{t}_{\mathrm{R}}($ anti major $)=51.8 \mathrm{~min}, \mathrm{t}_{\mathrm{R}}($ syn $)=59.6 \mathrm{~min}, \mathrm{t}_{\mathrm{R}}($ syn $)=$ $70.2 \mathrm{~min}$.
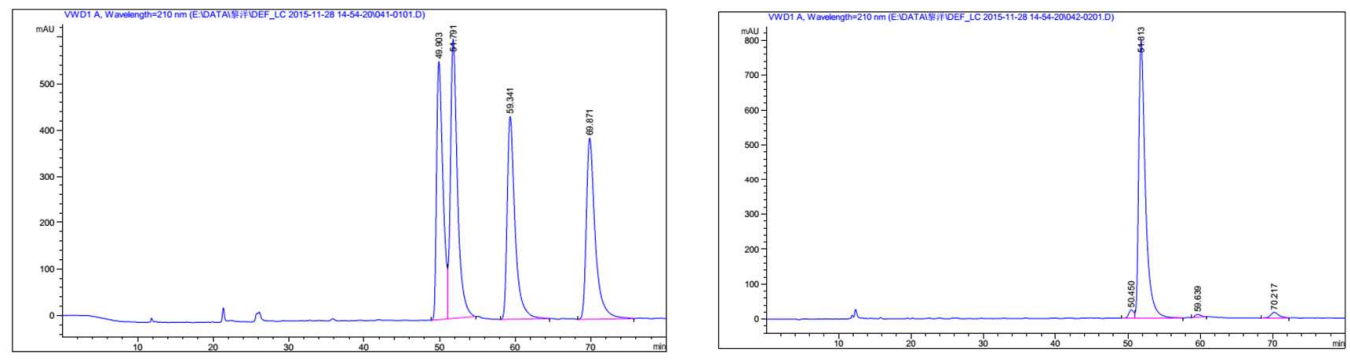

\begin{tabular}{llll}
\hline Peak\# & Retention Time & Area & Area\% \\
\hline 1 & 50.5 & 1102 & 2.109 \\
2 & 51.8 & 51156 & 97.891 \\
Total & & 52258 & 100.000 \\
\hline
\end{tabular}


$\overbrace{4 c a}^{O}$

Yellow oil in 99\% yield, $49.3 \mathrm{mg}, 11: 1 \mathrm{dr}$ and $92 \%$ ee. ${ }^{1} \mathrm{H}$ NMR (500 $\left.\mathrm{MHz}, \mathrm{CDCl}_{3}\right) \delta$ 7.60-7.56 (m, 2H), 7.46-7.41 (m, 2H), 5.41 (d, 0.98H, $J=$ 3.3, anti), $5.02(\mathrm{~d}, 0.09 \mathrm{H}, J=8.8$, syn $), 4.71-4.66(\mathrm{~m}, 0.09 \mathrm{H}$, syn),4.65-4.58 (m, 0.98H, anti), 3.08 (brs, 1H), 1.40 (d, 3H, $J=6.9)$.

HPLC (CHIRALCEL AD-H column, hexane/EtOH $=90 / 10$, flow $1 \mathrm{~mL} / \mathrm{min}$, detection at $210 \mathrm{~nm}$ ): $\mathrm{t}_{\mathrm{R}}($ anti minor $)=7.8 \mathrm{~min}, \mathrm{t}_{\mathrm{R}}($ anti major $)=10.4 \mathrm{~min}, \mathrm{t}_{\mathrm{R}}($ syn $)=11.2 \mathrm{~min}, \mathrm{t}_{\mathrm{R}}($ syn $)=18.8 \min$.
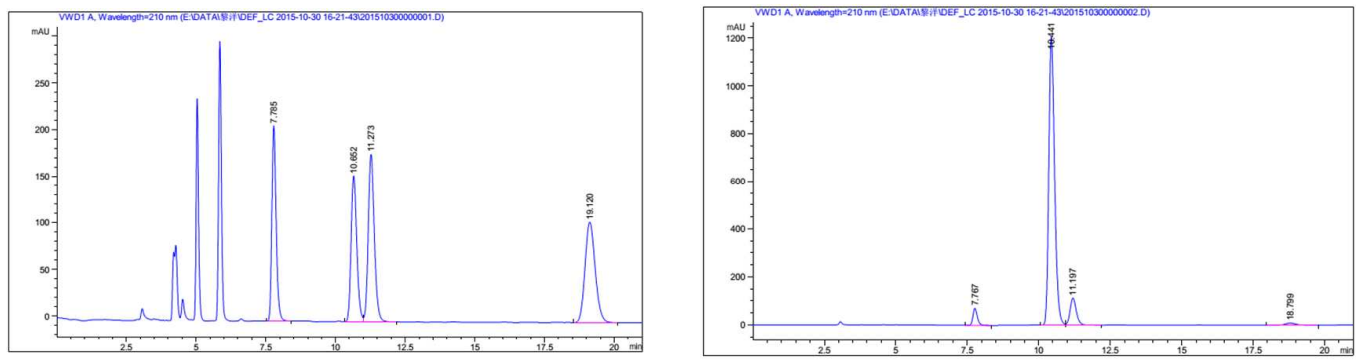

\begin{tabular}{llll}
\hline Peak\# & Retention Time & Area & Area $\%$ \\
\hline 1 & 7.8 & 756 & 4.234 \\
2 & 10.4 & 17098 & 95.766 \\
Total & & 17854 & 100.000 \\
\hline
\end{tabular}

Yellow oil in 99\% yield, $39.4 \mathrm{mg}, 14: 1 \mathrm{dr}$ and $92 \%$ ee. ${ }^{1} \mathrm{H}$ NMR $(500 \mathrm{MHz}$, $\left.\mathrm{CDCl}_{3}\right) \delta 7.31-7.27(\mathrm{~m}, 2 \mathrm{H}), 7.03-6.98(\mathrm{~m}, 2 \mathrm{H}), 5.29(\mathrm{~d}, 0.99 \mathrm{H}, J=3.6$, anti), $4.95(\mathrm{~d}, 0.07 \mathrm{H}, J=9.0$, syn $), 4.69-4.62(\mathrm{~m}, 0.07 \mathrm{H}$, syn $), 4.62-4.56(\mathrm{~m}$, 0.99H, anti), 2.71 (brs, 1H), $1.43(\mathrm{~d}, 3 \mathrm{H}, J=6.8)$. HPLC (CHIRALCEL AD-H column, hexane $/ \mathrm{EtOH}=90 / 10$, flow $1 \mathrm{~mL} / \mathrm{min}$, detection at $210 \mathrm{~nm}) \mathrm{t}_{\mathrm{R}}$ (anti minor) $=10.7$ $\min , \mathrm{t}_{\mathrm{R}}($ anti major $)=12.6 \mathrm{~min}, \mathrm{t}_{\mathrm{R}}(\mathrm{syn})=13.2 \mathrm{~min}, \mathrm{t}_{\mathrm{R}}(\mathrm{syn})=15.2 \mathrm{~min}$.
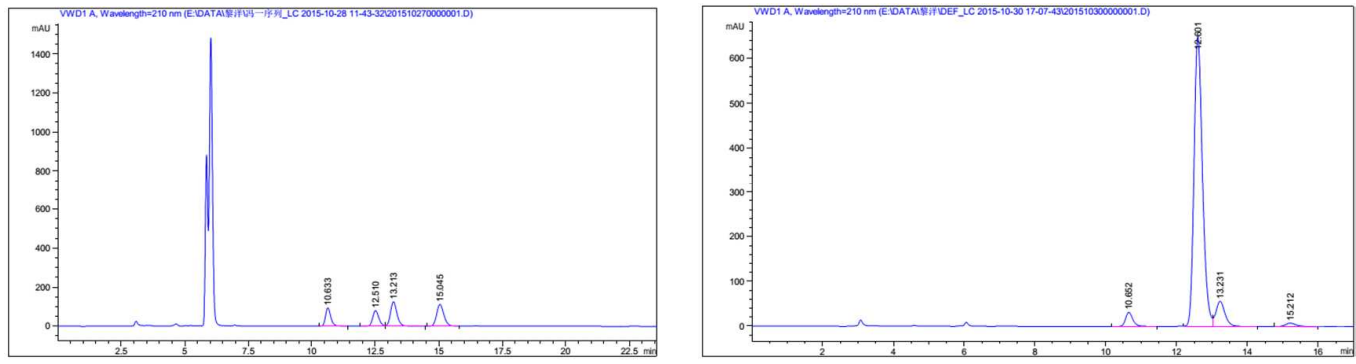


\begin{tabular}{llll}
\hline Peak\# & Retention Time & Area & Area\% \\
\hline 1 & 10.7 & 452 & 4.019 \\
2 & 12.6 & 10795 & 95.981 \\
Total & & 11247 & 100.000 \\
\hline
\end{tabular}

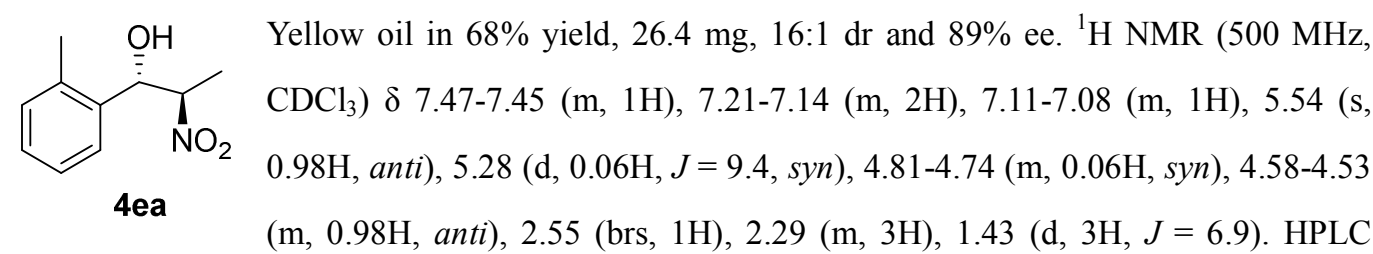

(CHIRALCEL AD-H column, hexane/2-propanol = 95/5, flow $1 \mathrm{~mL} / \mathrm{min}$, detection at $210 \mathrm{~nm}$ ): $\mathrm{t}_{\mathrm{R}}$ $($ anti major $)=10.2 \min , \mathrm{t}_{\mathrm{R}}($ anti minor $)=11.4 \min , \mathrm{t}_{\mathrm{R}}($ syn $)=13.8 \min , \mathrm{t}_{\mathrm{R}}($ syn $)=16.9 \min$.
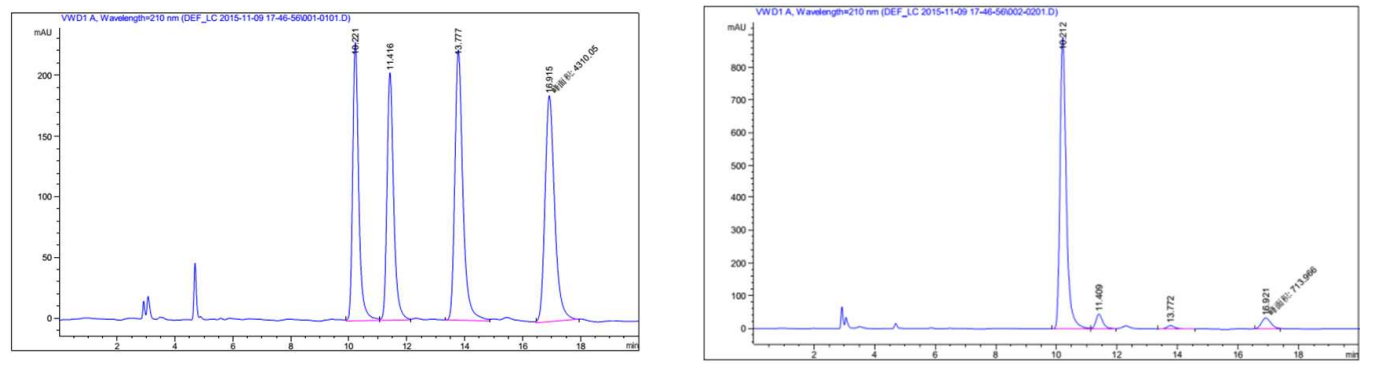

\begin{tabular}{llll}
\hline Peak\# & Retention Time & Area & Area\% \\
\hline 1 & 10.2 & 12810 & 94.588 \\
2 & 11.4 & 733 & 5.412 \\
Total & & 13543 & 100.000 \\
\hline
\end{tabular}

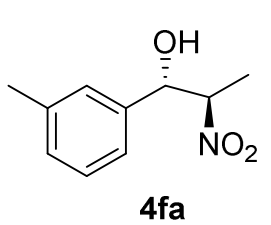

Yellow oil in $98 \%$ yield, $38.3 \mathrm{mg}, 24: 1 \mathrm{dr}$ and $96 \%$ ee. ${ }^{1} \mathrm{H}$ NMR $(500 \mathrm{MHz}$, $\left.\mathrm{CDCl}_{3}\right) \delta$ 7.21-7.17 (m, 1H), 7.11-7.05 (m, 3H), 5.27 (d, 0.96H, $J=3.6$, anti), $4.90(\mathrm{~d}, 0.04 \mathrm{H}, J=9.1, s y n), 4.72-4.65(\mathrm{~m}, 0.04 \mathrm{H}, s y n), 4.64-4.58(\mathrm{~m}, 0.96 \mathrm{H}$, anti), 2.65(brs, 1H), $2.29(\mathrm{~s}, 3 \mathrm{H}), 1.42(\mathrm{~d}, 3 \mathrm{H}, J=6.8)$. HPLC (CHIRALCEL

AS-H column, hexane/2-propanol $=90 / 10$, flow $1 \mathrm{~mL} / \mathrm{min}$, detection at $210 \mathrm{~nm}): \mathrm{t}_{\mathrm{R}}($ anti major $)=$ $8.8 \min , \mathrm{t}_{\mathrm{R}}($ anti $\operatorname{minor})=9.9 \min , \mathrm{t}_{\mathrm{R}}($ syn $)=10.6 \min , \mathrm{t}_{\mathrm{R}}(\operatorname{syn})=13.2 \min$. 

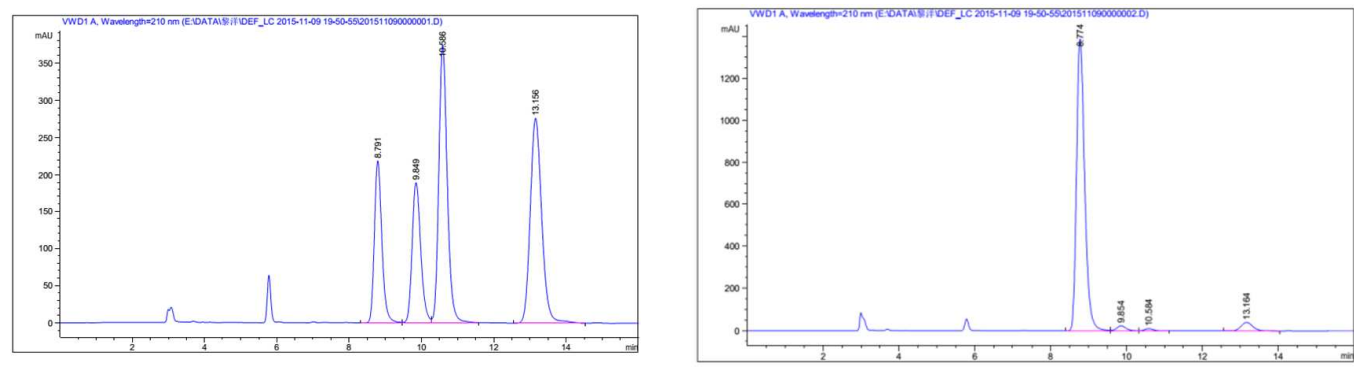

\begin{tabular}{llll}
\hline Peak\# & Retention Time & Area & Area\% \\
\hline 1 & 8.8 & 20646 & 98.039 \\
2 & 9.9 & 413 & 1.961 \\
Total & & 21059 & 100.000 \\
\hline
\end{tabular}

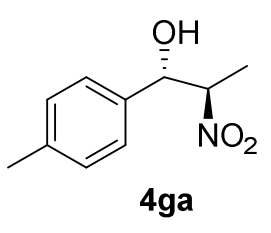

Yellow oil in 77\% yield, $30.2 \mathrm{mg}, 12: 1 \mathrm{dr}$ and 94\% ee. ${ }^{1} \mathrm{H}$ NMR (500 MHz, $\left.\mathrm{CDCl}_{3}\right) \delta 7.20-7.17(\mathrm{~m}, 2 \mathrm{H}), 7.14-7.10(\mathrm{~m}, 2 \mathrm{H}), 5.26(\mathrm{~d}, 0.86 \mathrm{H}, J=3.7$, anti), $4.91(\mathrm{~d}, 0.07 \mathrm{H}, J=9.1, s y n), 4.71-4.64(\mathrm{~m}, 0.07 \mathrm{H}, \mathrm{syn}), 4.63-4.57(\mathrm{~m}, 0.86 \mathrm{H}$, anti), 2.61 (brs, 1H), 2.28 (s, 3H), 1.44-1.42 (m, 3H). HPLC (CHIRALCEL

AD-H column, hexane $/ 2$-propanol $=95 / 5$, flow $1 \mathrm{~mL} / \mathrm{min}$, detection at $210 \mathrm{~nm}$ ): $\mathrm{t}_{\mathrm{R}}$ (anti major) $=$ $12.3 \min , \mathrm{t}_{\mathrm{R}}($ anti minor $)=14.2 \mathrm{~min}, \mathrm{t}_{\mathrm{R}}(s y n)=19.7 \mathrm{~min}, \mathrm{t}_{\mathrm{R}}(\mathrm{syn})=23.7 \mathrm{~min}$.
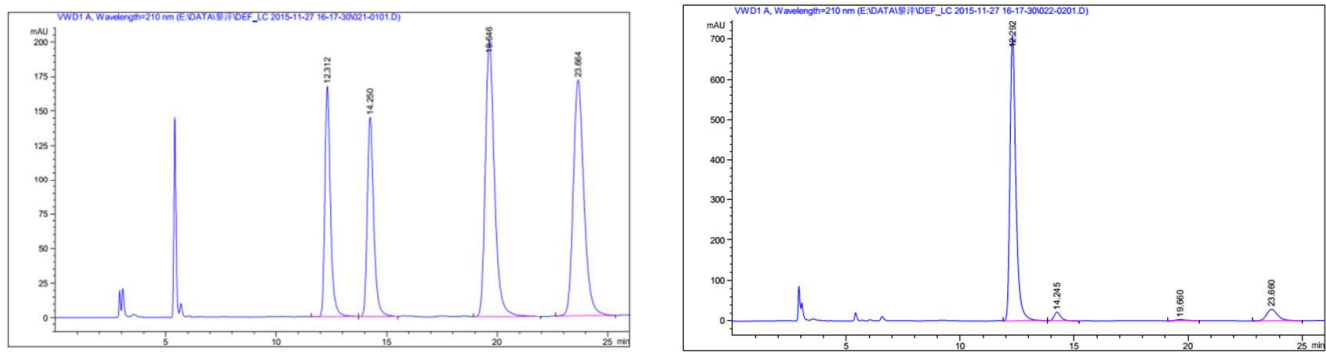

\begin{tabular}{llll}
\hline Peak\# & Retention Time & Area & Area\% \\
\hline 1 & 12.3 & 12666 & 96.761 \\
2 & 14.2 & 428 & 3.239 \\
Total & & 13094 & 100.000 \\
\hline
\end{tabular}

$\mathrm{OH} \quad$ Yellow oil in $96 \%$ yield, $32.7 \mathrm{mg}, 20: 1 \mathrm{dr}$ and $94 \%$ ee. ${ }^{1} \mathrm{H}$ NMR $(500 \mathrm{MHz}$,

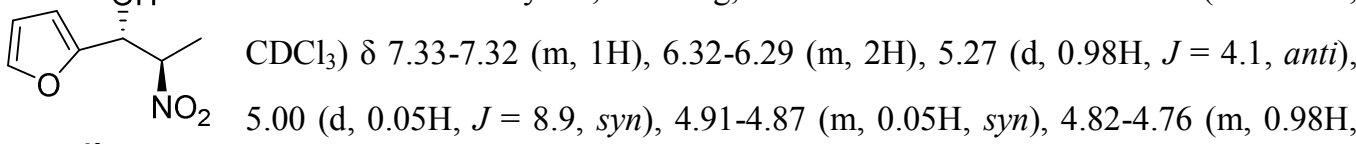
4ha anti), 2.70 (brs, 1H), $1.52(\mathrm{~d}, 3 \mathrm{H}, J=6.9)$. HPLC (CHIRALCEL OD-H + AD-H column, hexane $/ \mathrm{EtOH}=90 / 10$, flow $1 \mathrm{~mL} / \mathrm{min}$, detection at $210 \mathrm{~nm}): \mathrm{t}_{\mathrm{R}}($ anti minor $)=18.8 \mathrm{~min}$, 
$\mathrm{t}_{\mathrm{R}}($ syn $)=21.5 \mathrm{~min}, \mathrm{t}_{\mathrm{R}}($ anti major $)=22.4 \mathrm{~min}, \mathrm{t}_{\mathrm{R}}($ syn $)=23.8 \mathrm{~min}$.
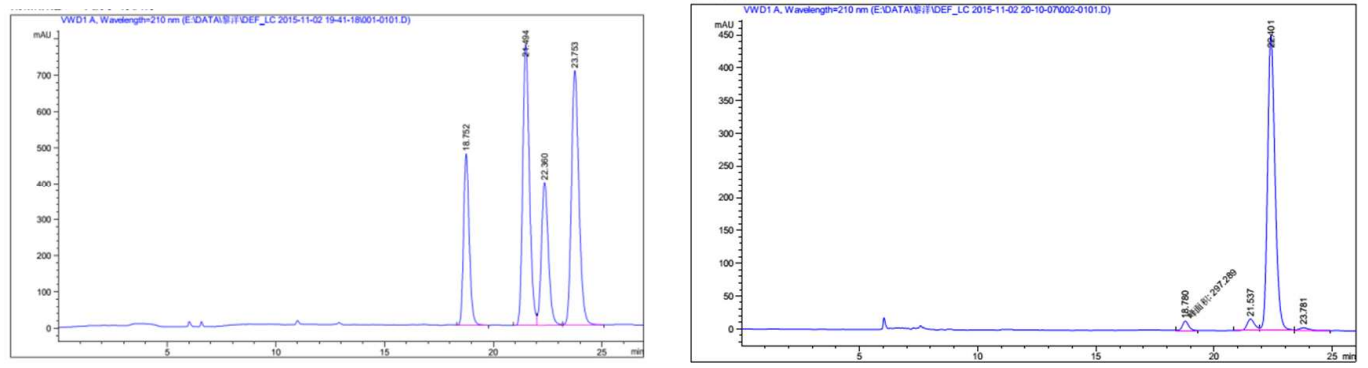

\begin{tabular}{llll}
\hline Peak\# & Retention Time & Area & Area\% \\
\hline 1 & 18.8 & 297 & 2.904 \\
2 & 22.4 & 9929 & 97.096 \\
Total & & 10226 & 100.000 \\
\hline
\end{tabular}

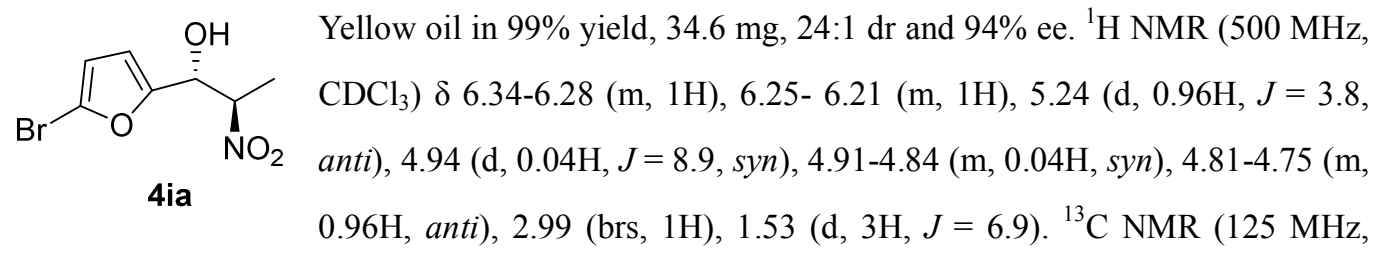
$\left.\mathrm{CDCl}_{3}\right) \delta 153.2,122.5,112.4,111.0,84.7,68.7,13.1$. HRMS (ESI): $\mathrm{m} / \mathrm{z}$ calcd for $\mathrm{C}_{7} \mathrm{H}_{7}{ }^{79} \mathrm{BrNO}_{4}$ [M-H] 247.95585, found 247.95513; calcd for $\mathrm{C}_{7} \mathrm{H}_{7}{ }^{81} \mathrm{BrNO}_{4}[\mathrm{M}-\mathrm{H}]^{-} 249.95380$, found 249.95307 . HPLC (CHIRALCEL AD-H column, hexane/EtOH = 95/5, flow $0.8 \mathrm{~mL} / \mathrm{min}$, detection at $210 \mathrm{~nm}$ ): $\mathrm{t}_{\mathrm{R}}($ anti major $)=18.9 \mathrm{~min}, \mathrm{t}_{\mathrm{R}}($ anti minor $)=19.9 \mathrm{~min}, \mathrm{t}_{\mathrm{R}}($ syn $)=22.8 \mathrm{~min}, \mathrm{t}_{\mathrm{R}}($ syn $)=27.5 \mathrm{~min}$.
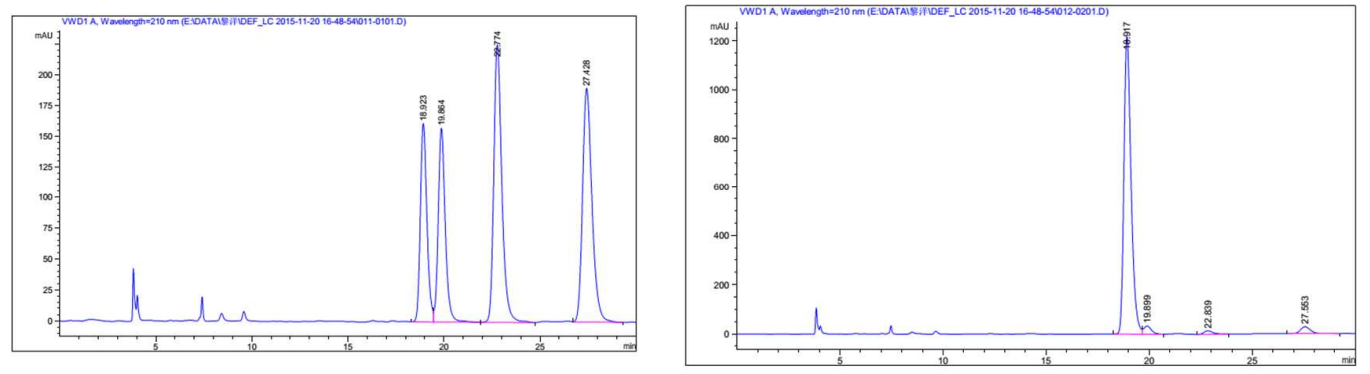

\begin{tabular}{llll}
\hline Peak\# & Retention Time & Area & Area\% \\
\hline 1 & 18.9 & 29265 & 97.155 \\
2 & 19.9 & 857 & 2.845 \\
Total & & 30122 & 100.000 \\
\hline
\end{tabular}




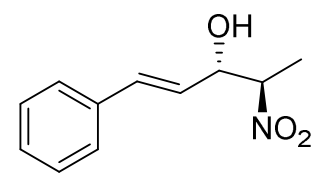

4ja

Yellow oil in 97\% yield, $40.0 \mathrm{mg},>30: 1 \mathrm{dr}$ and $98 \%$ ee. ${ }^{1} \mathrm{H}$ NMR (500 $\left.\mathrm{MHz}, \mathrm{CDCl}_{3}\right) \delta$ 7.32-7.18 (m, 5H), 6.69-6.65 (m, 1H), 6.07-6.02 (m, 1H), 4.80-4.78 (m, 0.99H anti), 4.61-4.55 (m, 1H, anti+syn), 2.54 (brs, 1H), $1.52(\mathrm{~d}, 3 \mathrm{H}, J=6.9)$. HPLC (CHIRALCEL AD-H column, hexane $/ 2$-propanol $=95 / 5$, flow $1 \mathrm{~mL} / \min$, detection at $210 \mathrm{~nm}): \mathrm{t}_{\mathrm{R}}($ anti major $)=20.6 \mathrm{~min}, \mathrm{t}_{\mathrm{R}}$ $($ anti $\operatorname{minor})=24.8 \mathrm{~min}, \mathrm{t}_{\mathrm{R}}(s y n)=25.9 \mathrm{~min}, \mathrm{t}_{\mathrm{R}}(\mathrm{syn})=33.1 \mathrm{~min}$.
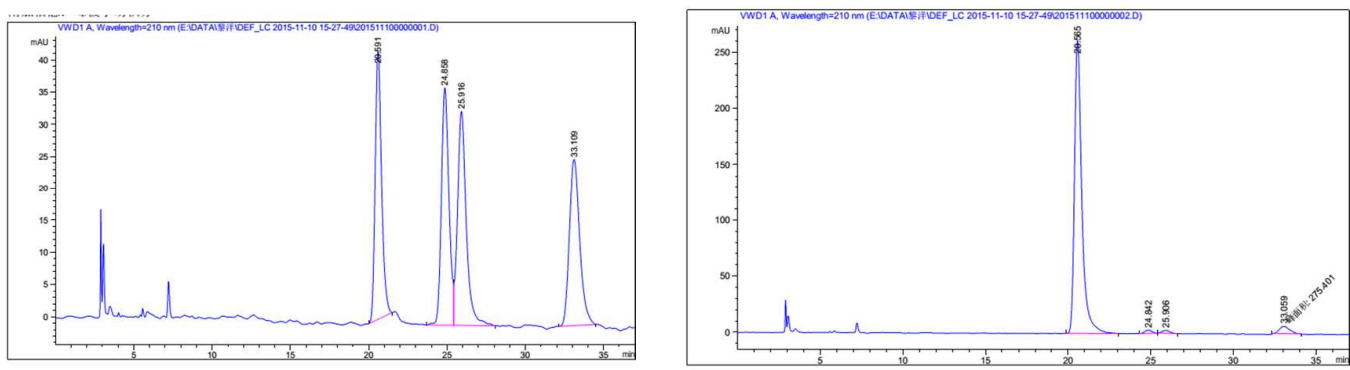

\begin{tabular}{llll}
\hline Peak\# & Retention Time & Area & Area\% \\
\hline 1 & 20.6 & 8076 & 98.971 \\
2 & 24.8 & 84 & 1.029 \\
Total & & 8160 & 100.000 \\
\hline
\end{tabular}

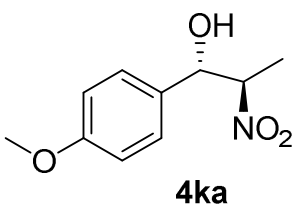

Yellow oil in $68 \%$ yield, $28.7 \mathrm{mg}, 11: 1 \mathrm{dr}$ and $92 \%$ ee. ${ }^{1} \mathrm{H}$ NMR $(500$ $\left.\mathrm{MHz}, \mathrm{CDCl}_{3}\right) \delta$ 7.24-7.20 (m, 2H), 6.87-6.81 (m, 2H), $5.23(\mathrm{~d}, 0.98 \mathrm{H}, J=$ 4.0, anti), $4.91(\mathrm{~d}, 0.09 \mathrm{H}, J=9.1$, syn $), 4.71-4.64(\mathrm{~m}, 0.09 \mathrm{H}$, syn), 4.62-4.56 (m, 0.98H, anti), $3.74(\mathrm{~s}, 3 \mathrm{H}), 2.57$ (brs, $1 \mathrm{H}), 1.45(\mathrm{~d}, 3 \mathrm{H}, J=$ 6.8). HPLC (CHIRALCEL AD-H column, hexane / 2-propanol = 90/10, flow $1 \mathrm{~mL} / \mathrm{min}$, detection at $210 \mathrm{~nm}): \mathrm{t}_{\mathrm{R}}($ anti major $)=11.2 \mathrm{~min}, \mathrm{t}_{\mathrm{R}}($ anti minor $)=12.7 \mathrm{~min}, \mathrm{t}_{\mathrm{R}}(\mathrm{syn})=16.0 \mathrm{~min}, \mathrm{t}_{\mathrm{R}}(\mathrm{syn})$ $=18.1 \mathrm{~min}$.
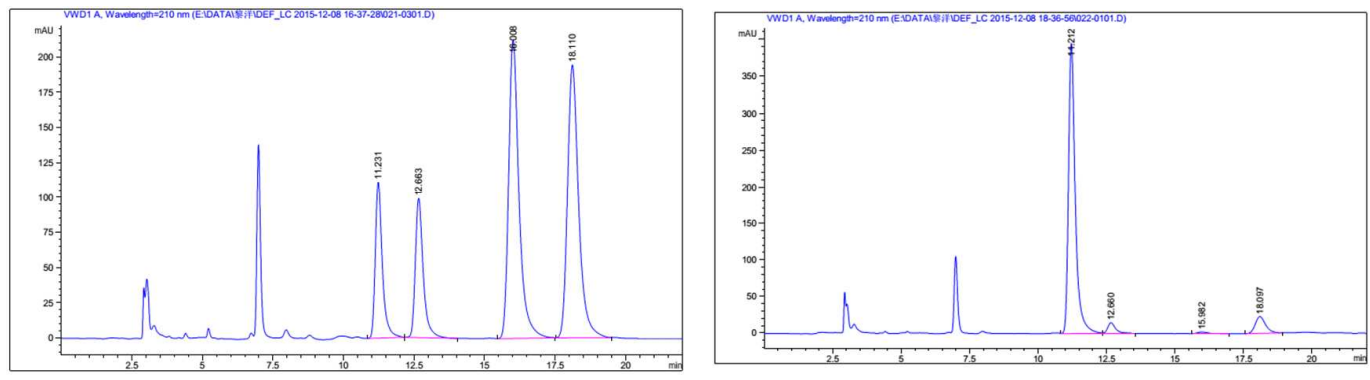


\begin{tabular}{llll}
\hline Peak\# & Retention Time & Area & Area\% \\
\hline 1 & 11.2 & 6814 & 95.877 \\
2 & 12.7 & 293 & 4.123 \\
Total & & 7107 & 100.000 \\
\hline
\end{tabular}

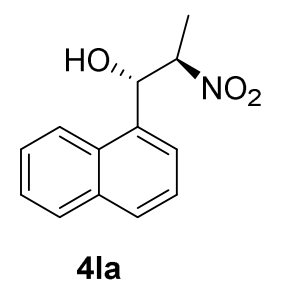

Yellow oil in 93\% yield, $43.1 \mathrm{mg}, 24: 1 \mathrm{dr}$ and $88 \%$ ee. ${ }^{1} \mathrm{H}$ NMR $(500 \mathrm{MHz}$, $\left.\mathrm{CDCl}_{3}\right) \delta 8.00(\mathrm{~d}, 1 \mathrm{H}, J=8.5), 7.90(\mathrm{~d}, 1 \mathrm{H}, J=8.1), 7.84(\mathrm{~d}, 1 \mathrm{H}, J=8.2)$, 7.79-7.77 (m, 1H), 7.62-7.57 (m, 1H), 7.55-7.50 (m, 2H), 6.28 (s, 0.95H, anti), 5.79 (d, 0.04H, $J=10.0, s y n), 5.16-5.09$ (m, 0.04H, syn), 4.94-4.89 (m, 0.95H, anti), 2.77 (brs, 1H), $1.43(\mathrm{~d}, 3 \mathrm{H}, J=6.8)$. HPLC (CHIRALCEL AD-H column, hexane/2-propanol $=90 / 10$, flow $1 \mathrm{~mL} / \mathrm{min}$, detection at $210 \mathrm{~nm}): \mathrm{t}_{\mathrm{R}}($ anti major $)=8.0$ $\min , \mathrm{t}_{\mathrm{R}}($ anti minor $)=9.8 \mathrm{~min}, \mathrm{t}_{\mathrm{R}}($ syn $)=13.2 \mathrm{~min}, \mathrm{t}_{\mathrm{R}}($ syn $)=15.3 \mathrm{~min}$.
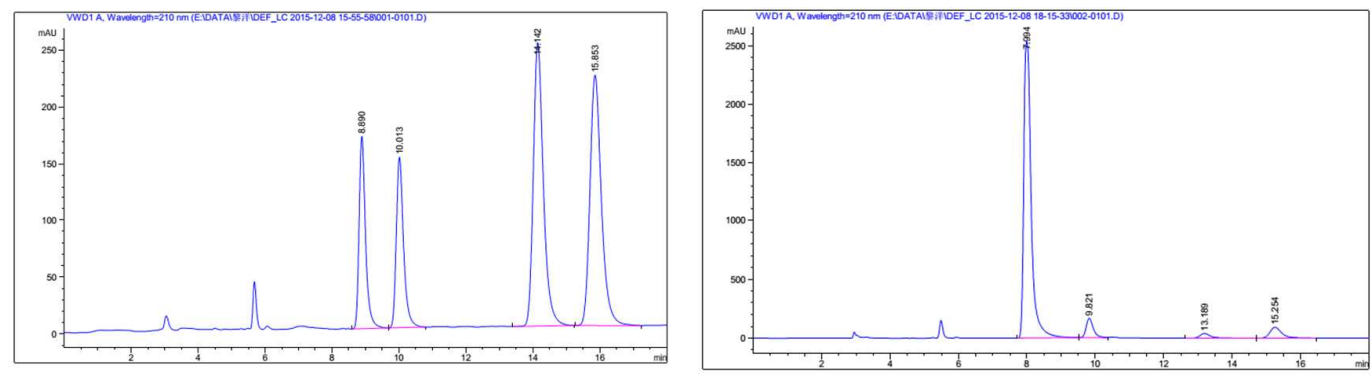

\begin{tabular}{llll}
\hline Peak\# & Retention Time & Area & Area\% \\
\hline 1 & 8.0 & 38075 & 94.040 \\
2 & 9.8 & 2413 & 5.960 \\
Total & & 40488 & 100.000 \\
\hline
\end{tabular}

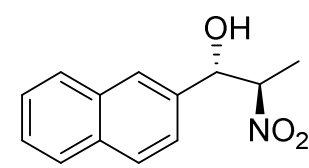

Yellow oil in 99\% yield, $45.9 \mathrm{mg}, 14: 1 \mathrm{dr}$ and $92 \%$ ee. ${ }^{1} \mathrm{H}$ NMR $(500$ $\left.\mathrm{MHz}, \mathrm{CDCl}_{3}\right) \delta$ 7.79-7.75 (m, 4H), 7.46-7.40 (m, 2H), 7.37-7.34 (m, 1H), $5.48(\mathrm{~d}, 1.00 \mathrm{H}, J=3.0$, anti), 5.09 (d, 0.07H, $J=9.1$, syn $), 4.83-4.76(\mathrm{~m}$, $4 \mathrm{ma}$ 0.07H, syn), 4.74-4.68 (m, 1.00H, anti), 2.82 (brs, $1 \mathrm{H}), 1.43$ (d, 3H, $J=$ 6.9). HPLC (CHIRALCEL AD-H column, hexane/2-propanol = 90/10, flow $1 \mathrm{~mL} / \mathrm{min}$, detection at $210 \mathrm{~nm}): \mathrm{t}_{\mathrm{R}}$ (anti major) $=10.5 \mathrm{~min}, \mathrm{t}_{\mathrm{R}}($ anti minor $)=12.4 \mathrm{~min}, \mathrm{t}_{\mathrm{R}}($ syn $)=16.7 \mathrm{~min}, \mathrm{t}_{\mathrm{R}}($ syn $)$ $=19.1 \mathrm{~min}$. 

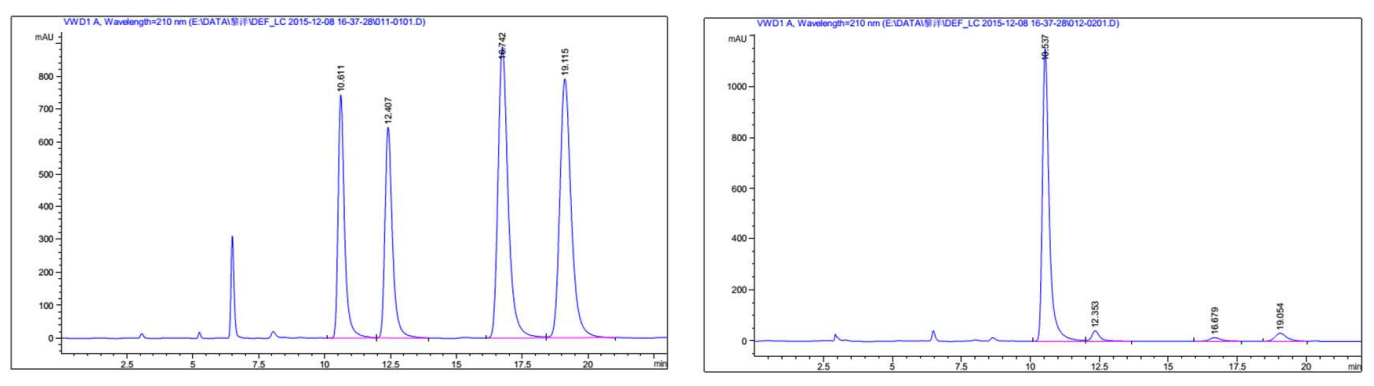

\begin{tabular}{llll}
\hline Peak\# & Retention Time & Area & Area\% \\
\hline 1 & 10.5 & 19696 & 95.970 \\
2 & 12.4 & 827 & 4.030 \\
Total & & 20523 & 100.000 \\
\hline
\end{tabular}

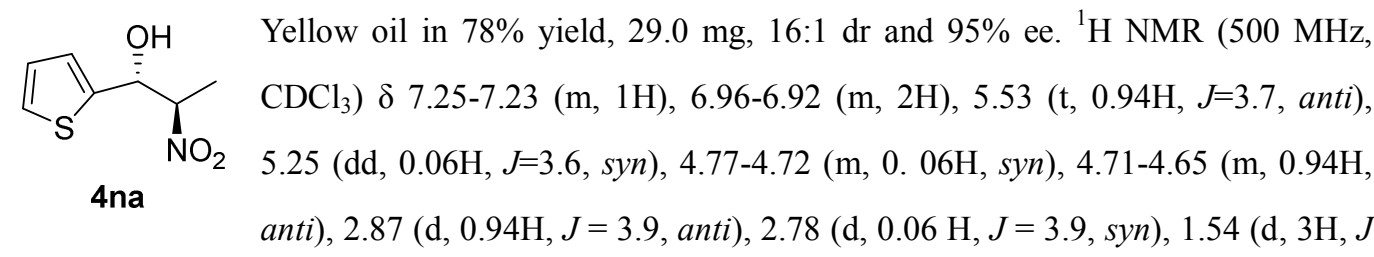
= 6.9). HPLC (CHIRALCEL AD-H column, hexane/EtOH $=90 / 10$, flow $1 \mathrm{~mL} / \mathrm{min}$, detection at $210 \mathrm{~nm}): \mathrm{t}_{\mathrm{R}}($ anti minor $)=13.4 \mathrm{~min}, \mathrm{t}_{\mathrm{R}}($ anti major $)=14.6 \mathrm{~min}, \mathrm{t}_{\mathrm{R}}($ syn $)=15.8 \min , \mathrm{t}_{\mathrm{R}}($ syn $)=16.7$ $\min$.
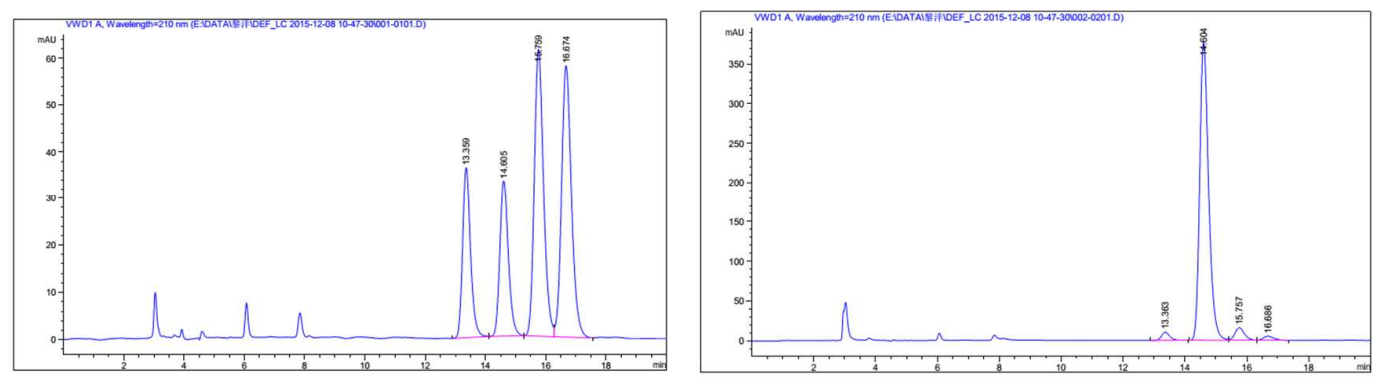

\begin{tabular}{llll}
\hline Peak\# & Retention Time & Area & Area\% \\
\hline 1 & 13.4 & 184 & 2.394 \\
2 & 14.6 & 7501 & 97.606 \\
Total & & 7685 & 100.000 \\
\hline
\end{tabular}




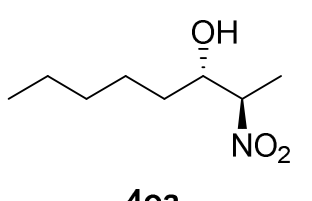

Yellow oil in 54\% yield, $18.9 \mathrm{mg}, 3: 1 \mathrm{dr}$ and 85\% ee. ${ }^{1} \mathrm{H}$ NMR $(500 \mathrm{MHz}$, $\left.\mathrm{CDCl}_{3}\right) \delta 4.49-4.41(\mathrm{~m}, 0.93 \mathrm{H}$, anti + syn $), 4.13-4.09(\mathrm{~m}, 0.70 \mathrm{H}$, anti), 3.85-3.81 (m, 0.23H, syn), 2.30 (brs, $1 \mathrm{H}), 1.50-1.47$ (m, 3H), 1.39-1.16 (m, 8H), 0.85-0.80 (m, 3H). HPLC (CHIRALCEL AD-H column, hexane $/ \mathrm{EtOH}=90 / 10$, flow $1 \mathrm{~mL} / \mathrm{min}$, detection at $210 \mathrm{~nm}): \mathrm{t}_{\mathrm{R}}($ anti minor $)=8.7 \mathrm{~min}, \mathrm{t}_{\mathrm{R}}($ anti major $)=9.5 \min , \mathrm{t}_{\mathrm{R}}(s y n)=10.2 \mathrm{~min}, \mathrm{t}_{\mathrm{R}}(s y n)=11.9 \mathrm{~min}$.
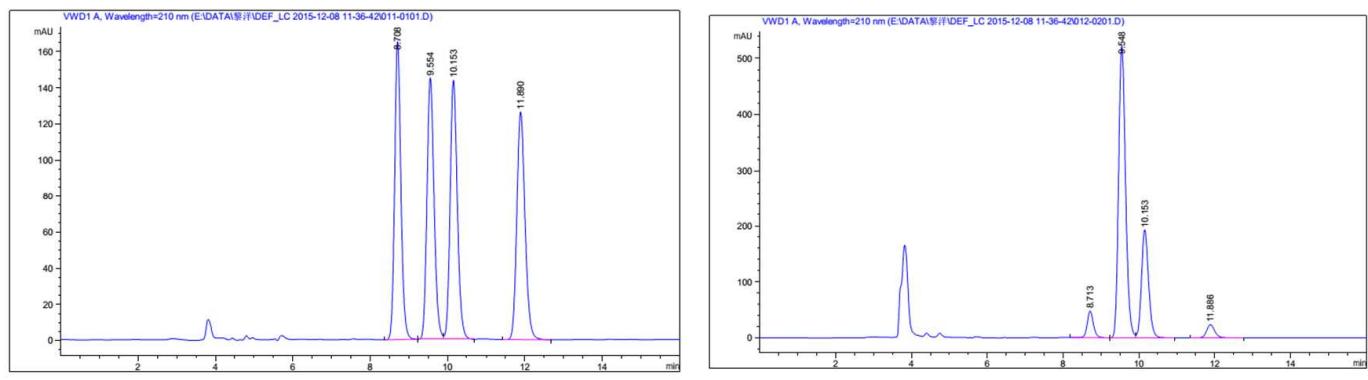

\begin{tabular}{llll}
\hline Peak\# & Retention Time & Area & Area\% \\
\hline 1 & 8.7 & 538 & 7.459 \\
2 & 9.5 & 6675 & 92.541 \\
Total & & 7213 & 100.000 \\
\hline
\end{tabular}

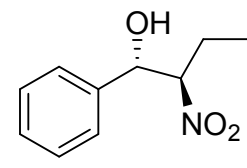

$4 a b$

Yellow oil in 56\% yield, $21.7 \mathrm{mg}, 20: 1 \mathrm{dr}$ and $86 \%$ ee. ${ }^{1} \mathrm{H}$ NMR $(500 \mathrm{MHz}$, $\left.\mathrm{CDCl}_{3}\right) \delta 7.33-7.24(\mathrm{~m}, 5 \mathrm{H}), 5.10(\mathrm{~d}, 0.98 \mathrm{H}, J=4.9$, anti), $4.95(\mathrm{~d}, 0.05 \mathrm{H}, J=$ 9.1, syn), 4.57-4.54 (m, 0.05H, syn), 4.53 - 4.48 (m, 0.98H, anti), 2.63 (brs, $1 \mathrm{H}), 2.15-2.04(\mathrm{~m}, 1 \mathrm{H}), 1.88-1.79(\mathrm{~m}, 1 \mathrm{H}), 0.86(\mathrm{t}, 3 \mathrm{H}, J=7.4)$. HPLC (CHIRALCEL AD-H column, hexane/EtOH = 95/5, flow $1 \mathrm{~mL} / \mathrm{min}$, detection at $210 \mathrm{~nm}$ ): $\mathrm{t}_{\mathrm{R}}$ $($ anti $\operatorname{minor})=14.2, \mathrm{t}_{\mathrm{R}}($ anti major $)=17.8 \mathrm{~min}, \mathrm{t}_{\mathrm{R}}($ syn $)=19.0 \mathrm{~min}, \mathrm{t}_{\mathrm{R}}(\mathrm{syn})=25.2 \mathrm{~min}$.
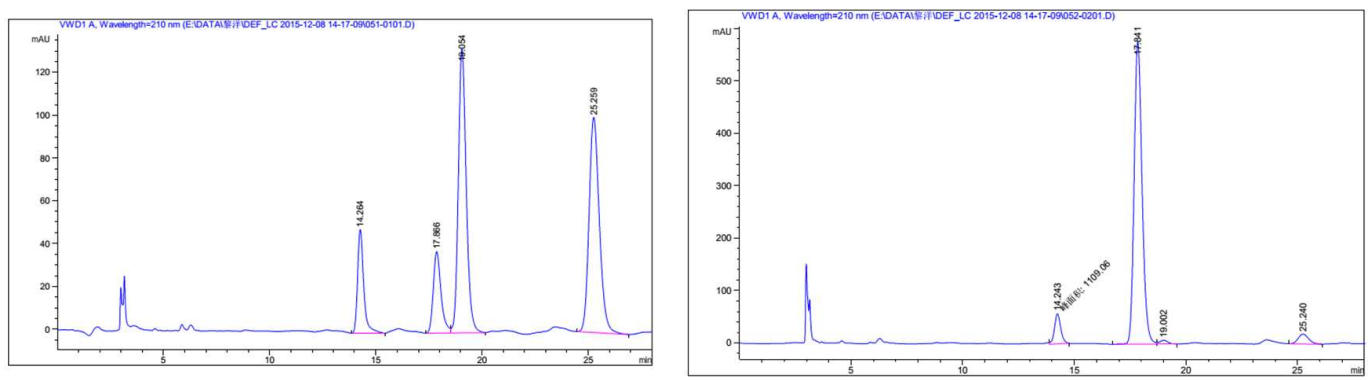


\begin{tabular}{llll}
\hline Peak\# & Retention Time & Area & Area\% \\
\hline 1 & 14.2 & 1109 & 7.234 \\
2 & 17.8 & 14221 & 92.766 \\
Total & & 15330 & 100.000 \\
\hline
\end{tabular}

\section{Miscellaneous analyses of the $\mathrm{Cu} / \mathrm{Sm} / 1$ complex}

\subsection{ESI-MS analysis of the complexes}

Instrument: Waters Acquity SQ Detector

Soure mode: ESI (Electrospray ionization)

Conditions: Capillary (kV) 3.00(+); Cone (V) 30.00; Extractor (V) 0.00; RF (V) 0.00; Source Temperature $\left({ }^{\circ} \mathrm{C}\right)$ 100; Desolvation Temperature $\left({ }^{\circ} \mathrm{C}\right) \quad 350 ;$ Cone Gas Flow $\quad(\mathrm{L} / \mathrm{Hr}) \quad 55$; Desolvation Gas Flow (L/Hr) 550.

Sample preparation: $\mathrm{Cu}(\mathrm{OAc})_{2}(0.02 \mathrm{mmol}), \mathrm{Sm}(\mathrm{O}-i \mathrm{Pr})_{3}(0.02 \mathrm{mmol}), 1(0.02 \mathrm{mmol})$ and $i \mathrm{Pr}_{2} \mathrm{NEt}$ $(0.06 \mathrm{mmol})$ were stirred in THF $(1.0 \mathrm{~mL})$ by the one-pot procedure at $35^{\circ} \mathrm{C}$ for $2 \mathrm{~h}$. The mixture was diluted with $\mathrm{CH}_{3} \mathrm{CN}$ and injected for analysis.

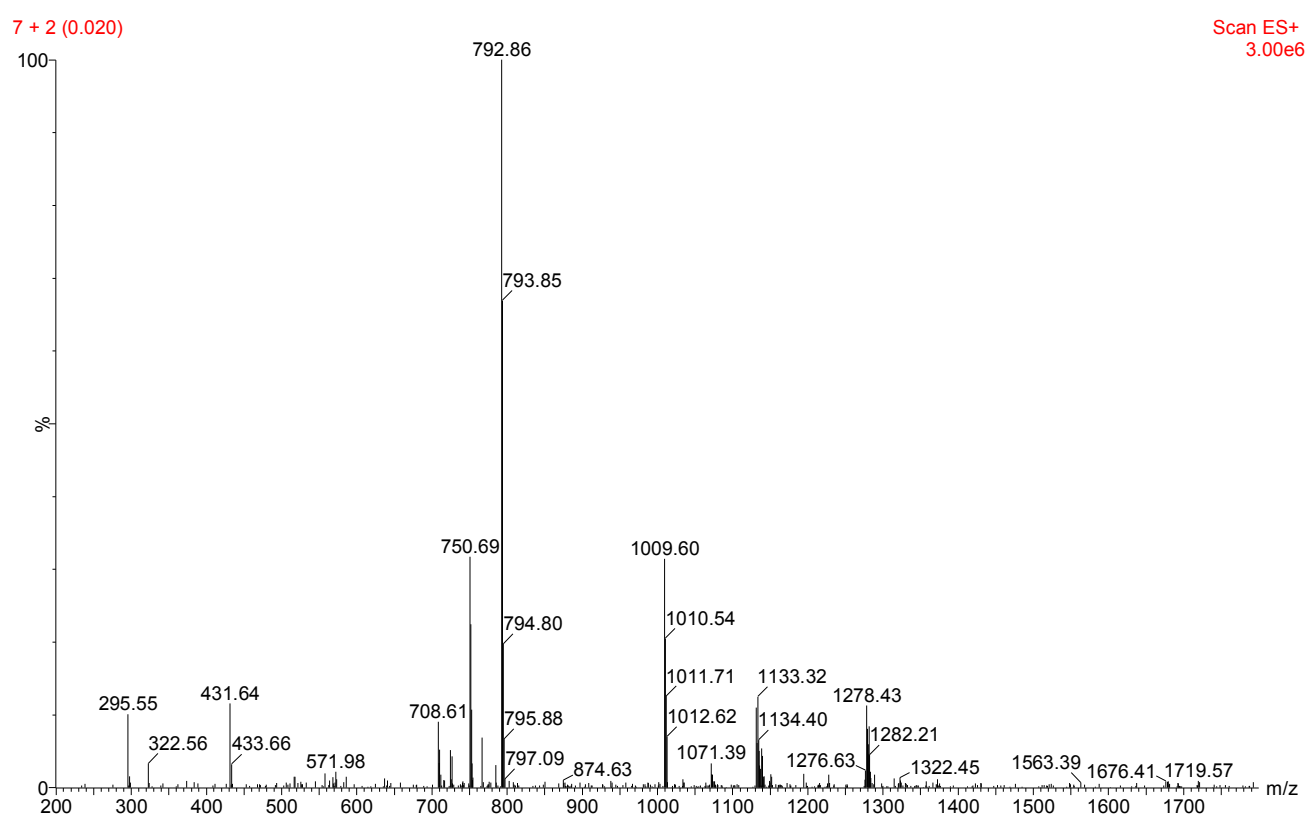




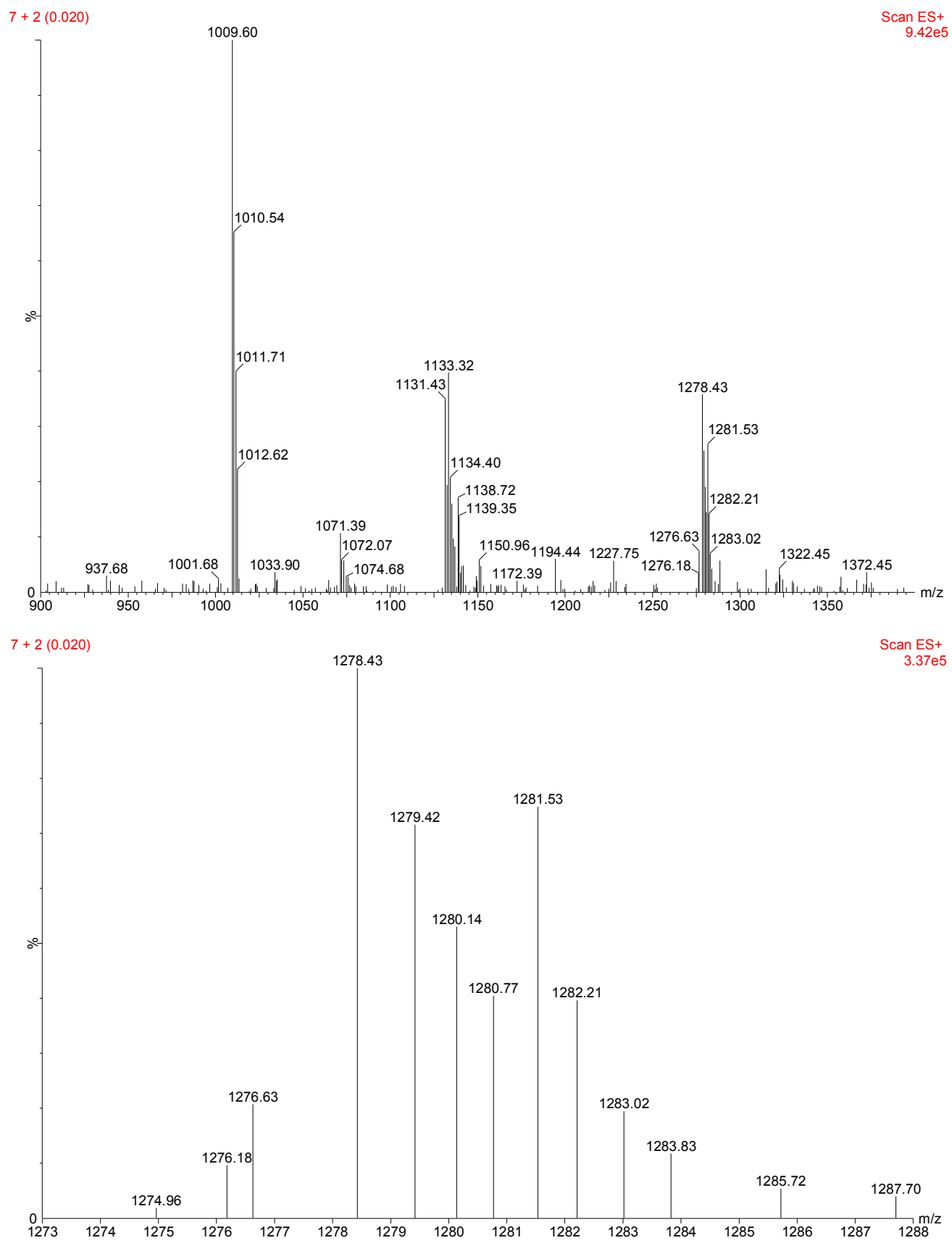

Figure S1. ESI-MS of $\mathrm{Cu} / \mathrm{Sm} / \mathbf{1}=1: 1: 1$ mixture with the additive of $i \mathrm{Pr}_{2} \mathrm{NEt}$

Calcd. For $\mathrm{C}_{63} \mathrm{H}_{49} \mathrm{Cu}_{2} \mathrm{~N}_{4} \mathrm{O}_{5} \mathrm{~S}_{2}$ [Ligand - $\left.\mathrm{H}_{3}+\mathrm{Cu}_{2}\right]^{+}:$1131.17; Found: 1131.43

Calcd. For $\mathrm{C}_{66} \mathrm{H}_{56} \mathrm{CuN}_{4} \mathrm{O}_{6} \mathrm{~S}_{2} \mathrm{Sm}$ [Ligand $\left.-\mathrm{H}_{3}+\mathrm{Cu}+\mathrm{SmOiPr}\right]^{+}: 1279.21$; Found:1279.42

In ESI-MS charts of $\mathrm{Cu} / \mathrm{Sm} / \mathbf{1}=1: 1: 1$ mixture with the additive of $i \operatorname{Pr}_{2} \mathrm{NEt}$ (Figure S1), the peaks corresponding to $\mathrm{Cu}_{2} / \mathbf{1}$ and $\mathrm{Cu} / \mathrm{Sm} / \mathbf{1}$ were detected. The peak of $\mathrm{m} / \mathrm{z}=1279$ was assigned to [Ligand $\left.-\mathrm{H}_{3}+\mathrm{Cu}+\mathrm{SmOiPr}\right]^{+}$rather than [Ligand $\left.-\mathrm{H}_{3}+\mathrm{CuOAc}+\mathrm{Sm}\right]^{+}$(Calcd. For $\mathrm{C}_{65} \mathrm{H}_{51} \mathrm{CuN}_{4} \mathrm{O}_{7} \mathrm{~S}_{2} \mathrm{Sm}$ [Ligand $\left.-\mathrm{H}_{3}+\mathrm{CuOAc}+\mathrm{Sm}\right]^{+}:$1279.18) according to the analysis of $\mathrm{m} / \mathrm{z}=$ 


\subsection{Discussion on the $\mathrm{Cu} / \mathrm{Sm} / 1$ complex}

6.2.1 For control experiments, see Table 1. Neither the $\mathrm{Cu}-\mathbf{1}$ complex nor the $\mathrm{Cu}_{2}-\mathbf{1}$ complex could catalyze the reaction, even in the presence of $30 \mathrm{~mol} \% i \operatorname{Pr} 2 \mathrm{NEt}$ (Entries 2 and 3, Table 1). The $\mathrm{Sm}_{\mathrm{x}}-\mathbf{1}_{\mathrm{y}}$ complexes could catalyze the reaction with modest anti-selectivity and racemic product (Entries 4 and 5, Table 1). The ratio of $\mathrm{Cu} / \mathrm{Sm} / \mathbf{1}$ in 1:1:1 was also critical for good selectivity (Entry 1 vs. entries 6 and 7, Table 1). On the basis of control experiments and the ESI-MS analysis, we assumed that a monomeric $\mathrm{Cu} / \mathrm{Sm} / 1=1: 1: 1$ complex would be the active species.

6.2.2 It was interesting that the addition order of metal salts could hardly affect the ee values of the anti-product; whereas inferior yield and dr were observed when reversing the addition order of $\mathrm{Cu}(\mathrm{OAc})_{2}$ and $\mathrm{Sm}(\mathrm{O}-i \mathrm{Pr})_{3}$ (Entry 9 vs entries 1 and 8, Table 1). On the basis of these results and the geometry of 1 optimized using density functional theory (DFT) at the B3LYP/ 6-31G (d) level (Figure $\mathrm{S} 2)^{[2]}$, we speculated that the $\mathrm{Cu}$ and $\mathrm{Sm}$ would incorporate with the ligand side by side. According to previous reports about the structures of Titanium complexes of $N$-sulfonylated $\beta$-amino alcohols ${ }^{[3]}$, as well as the optimized geometry of $\mathbf{1}$ (Figure S2), we assumed that the ligand could coordinate to the $\mathrm{Cu}$ or Sm via phenol oxygen, secondary amine nitrogens, sulfonamide oxygens and sulfonamide nitrogens. According to the ESI-MS analysis of the complex and the balance of total charge, four protons should be removed (Figure S3).
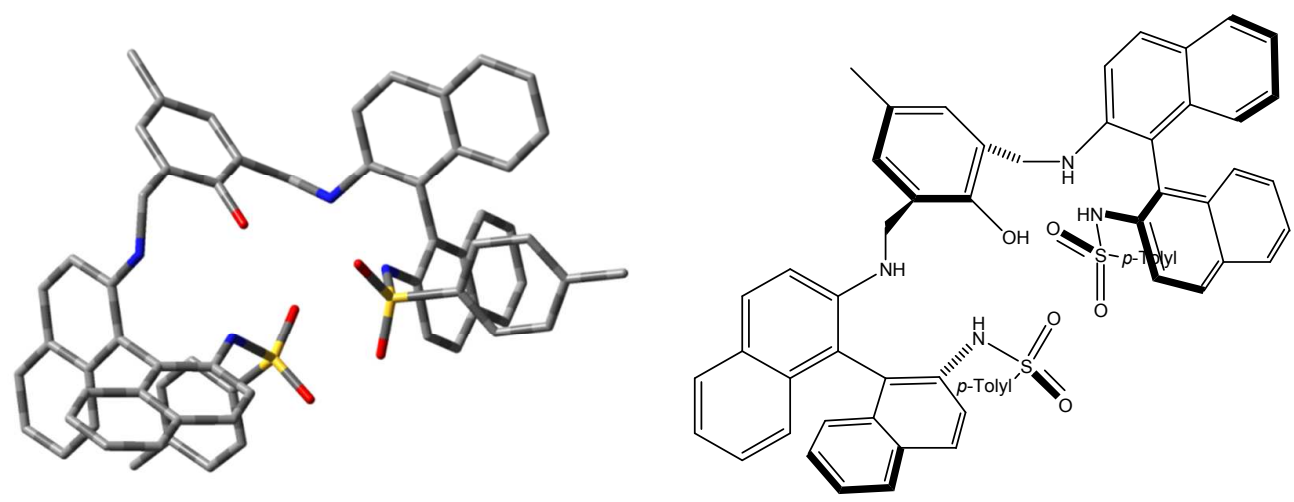

Figure S2. The geometry of 1 optimized using density functional theory (DFT) at the B3LYP/ 6-31G (d) level

[2] The calculations were performed using the Gaussian 09 program package.

[3]. The structures of related Titanium complexes of $N$-sulfonylated $\beta$-amino alcohols, see: (8a) Wu, K. H.; Gau, H. M.; Organometallics, 2003, 22, 5193-5200. (8b) Wu, K. H.; Gau, H. M.; Organometallics, 2004, 23, 580-588. (8c) Hsieh, S. H.; Gau, H. M. Chirality, 2006, 18, 569-574. 


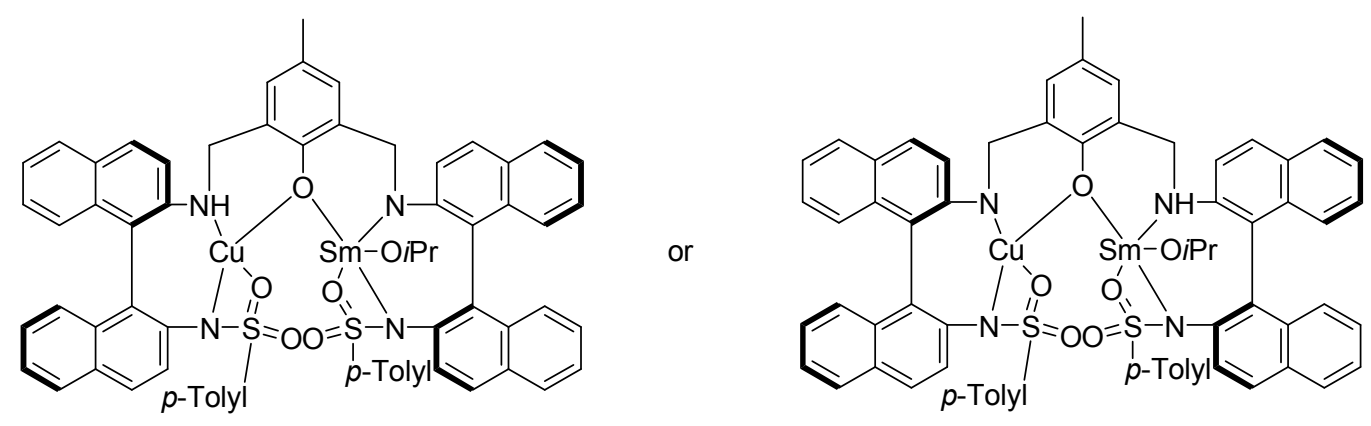

Figure S3. The proposed structure of heterobimetallic $\mathrm{Cu} / \mathrm{Sm} / \mathbf{1}$ complex

6.2.3 Based on the geometry of 1 optimized using density functional theory (DFT) at the B3LYP/ 6-31G (d) level and the absolute configuration of the products, four plausible transition states TS1-4 (Figure S4) were proposed to elucidate the asymmetric induction. As both $\mathrm{Cu}$ and $\mathrm{Sm}$ were essential in this catalytic system, we took the cooperative dual coordination of aldehydes and nitroalkanes into account. We assumed that the nitronate generated by $i \mathrm{Pr}_{2} \mathrm{NEt}$ would be orientated by $\mathrm{Sm}$ and the aldehyde would coordinate with $\mathrm{Cu}^{4}{ }^{4} \mathrm{TS1}$ was speculated to be more favorable than TS2-4 due to the steric repulsion between R (or R') and the $\mathrm{Cu} / \mathrm{Sm}$ complex, affording ( $1 \mathrm{~S}$, $2 R)-4$.

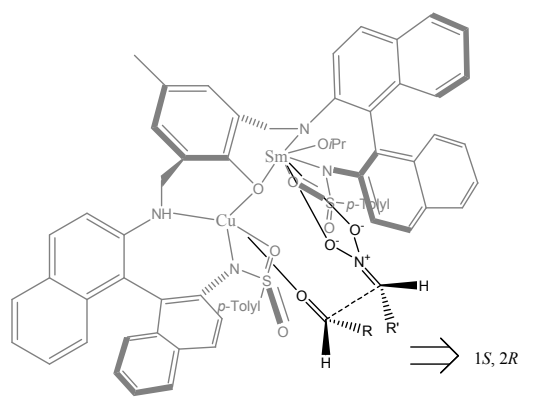

TS1 favored

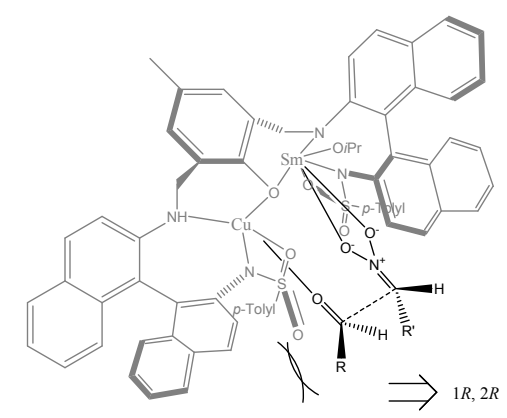

TS3 disfavored

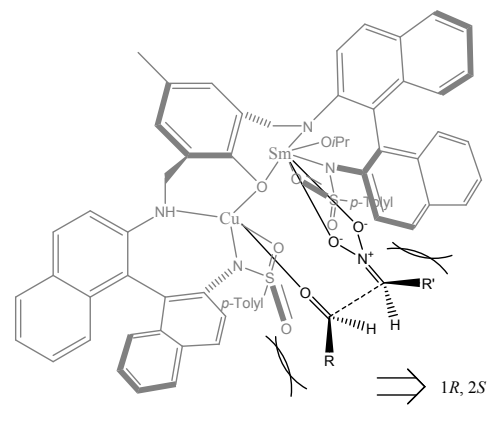

TS2 disfavored

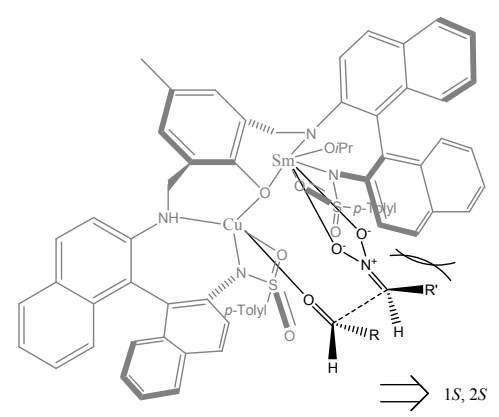

TS4 disfavored

Figure S4. The proposed working model

[4] (4g) Handa, S.; Gnanadesikan, V.; Matsunaga, S.; Shibasaki, M. J. Am. Chem. Soc. 2007, 129, 4900-4901. (4h) Handa, S.; Gnanadesikan, V.; Matsungasa, S.; Shibasaki, M. J. Am. Chem. Soc. 2010, 132, 4925-4934. 
6.2.4 There were two kinds of coordination in the mode of "side by side": A and B, while the main product was the same one according the proposed favored transition states (Figure S5). So there was almost no difference between $\mathrm{A}$ and $\mathrm{B}$ in this reaction.

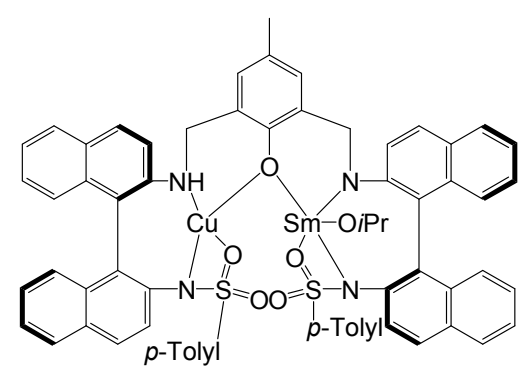

$(R, R)-\mathrm{Cu} / \mathrm{Sm} / \mathbf{1} \mathbf{A}$

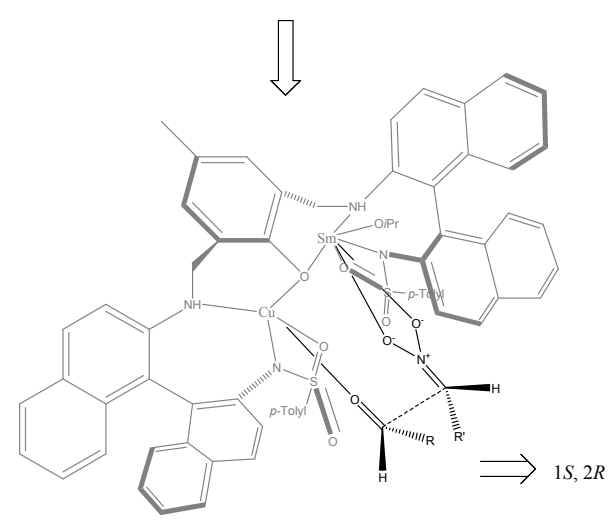

TS1 favored

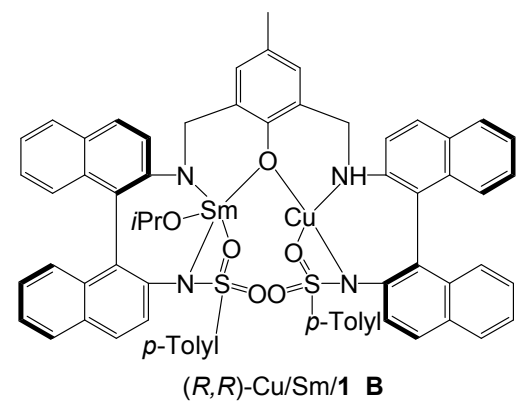

$\sqrt{1}$

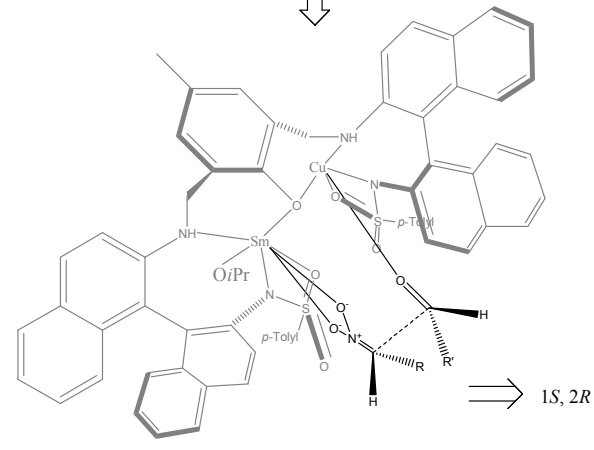

TS1-1 favored

Figure S5. Proposed structure of heterometallic complex and the favored transition states 


\section{Copies of NMR spectra}

$(R, R)-1$

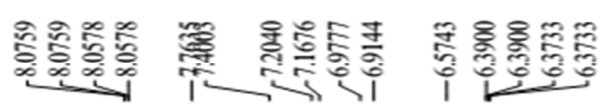

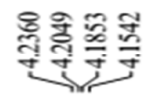

产

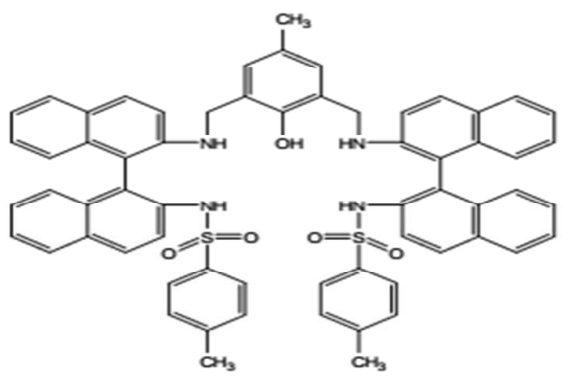

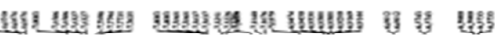
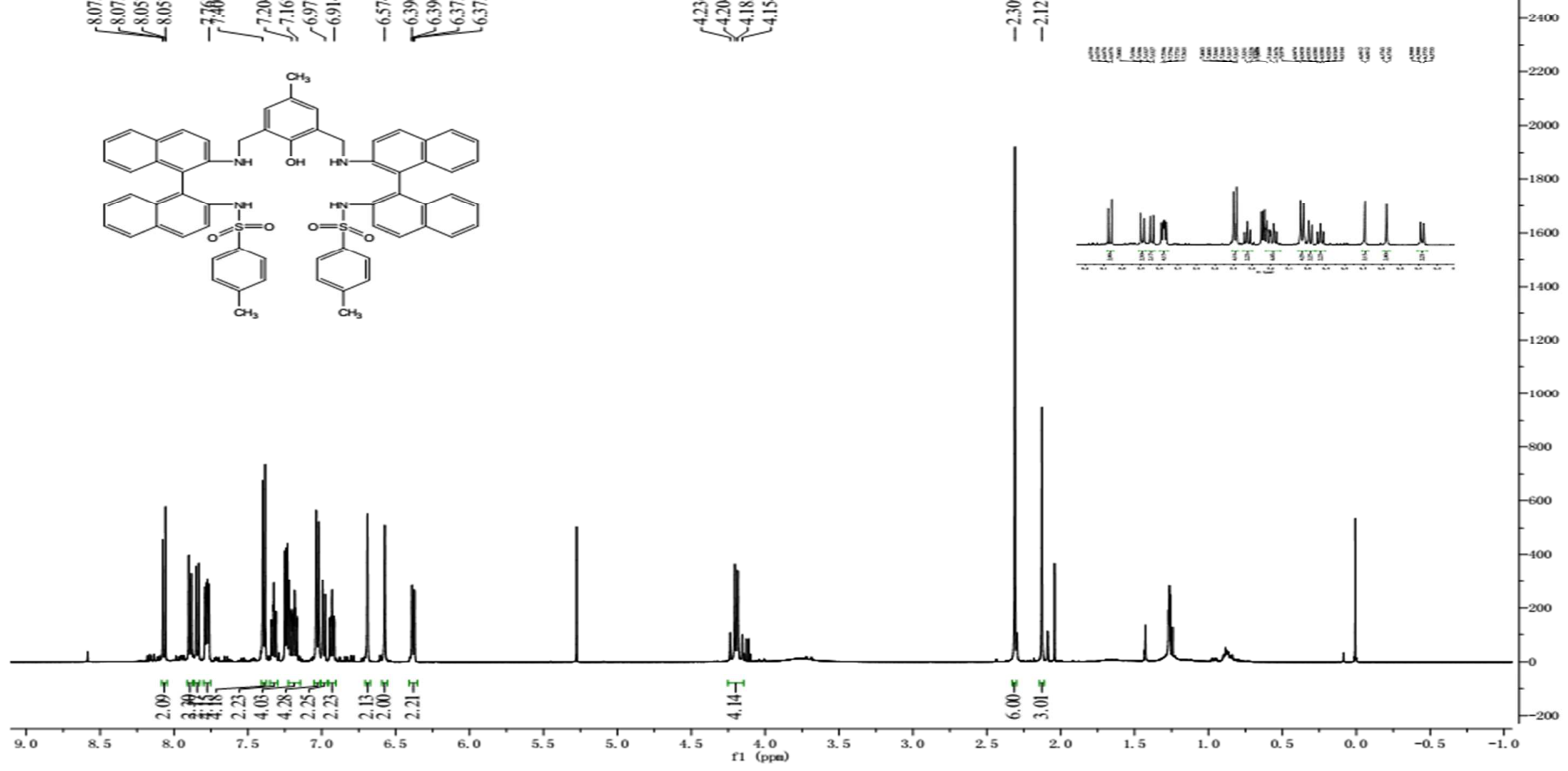
$(R, R)-1$

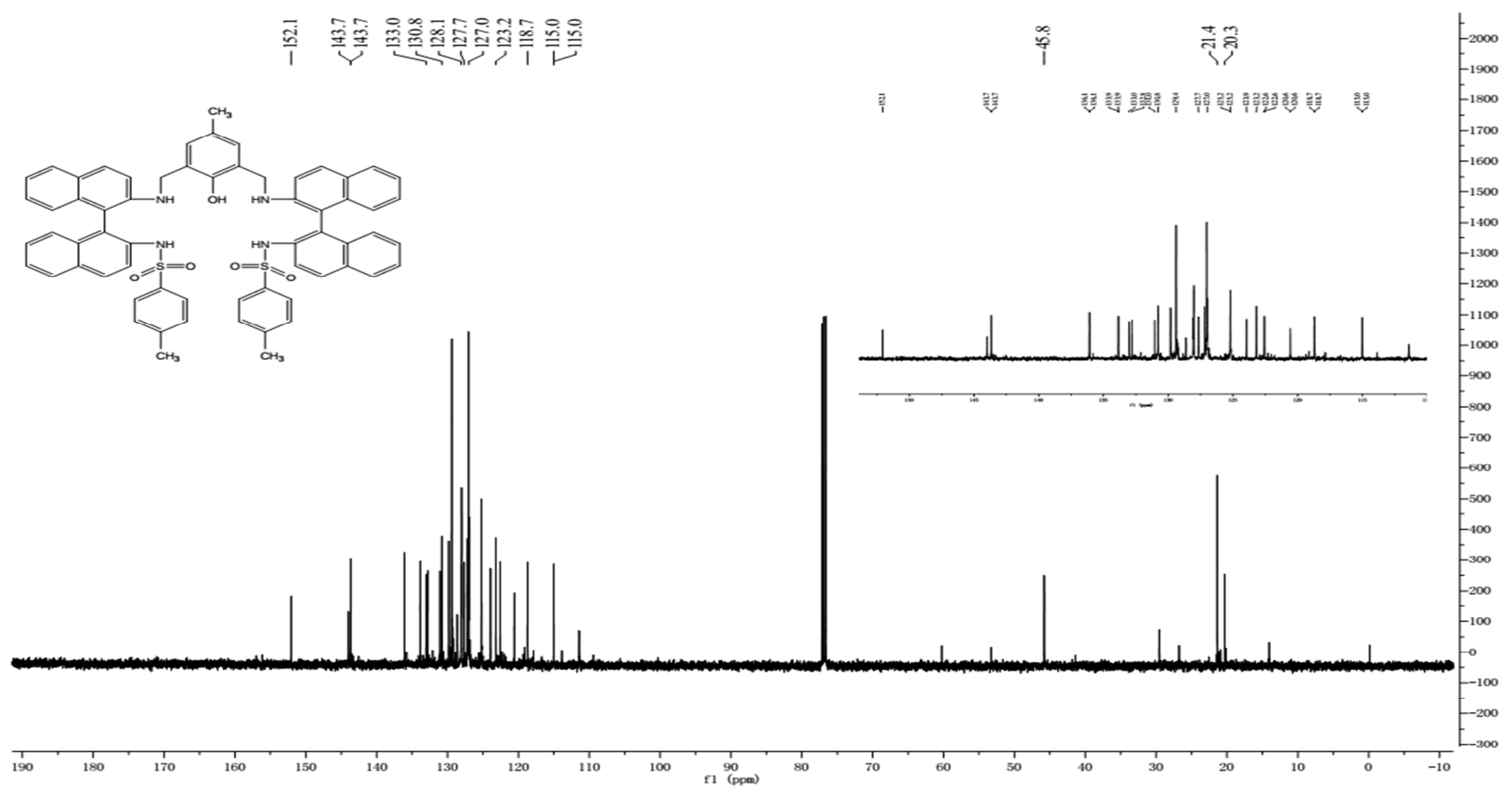


4aa (Entry 1, Table 2)

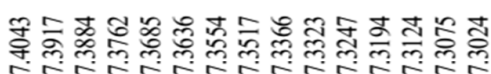

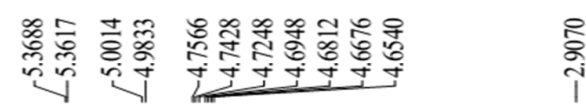

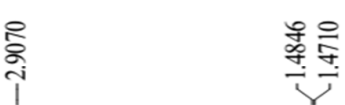

7500

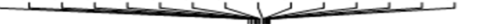
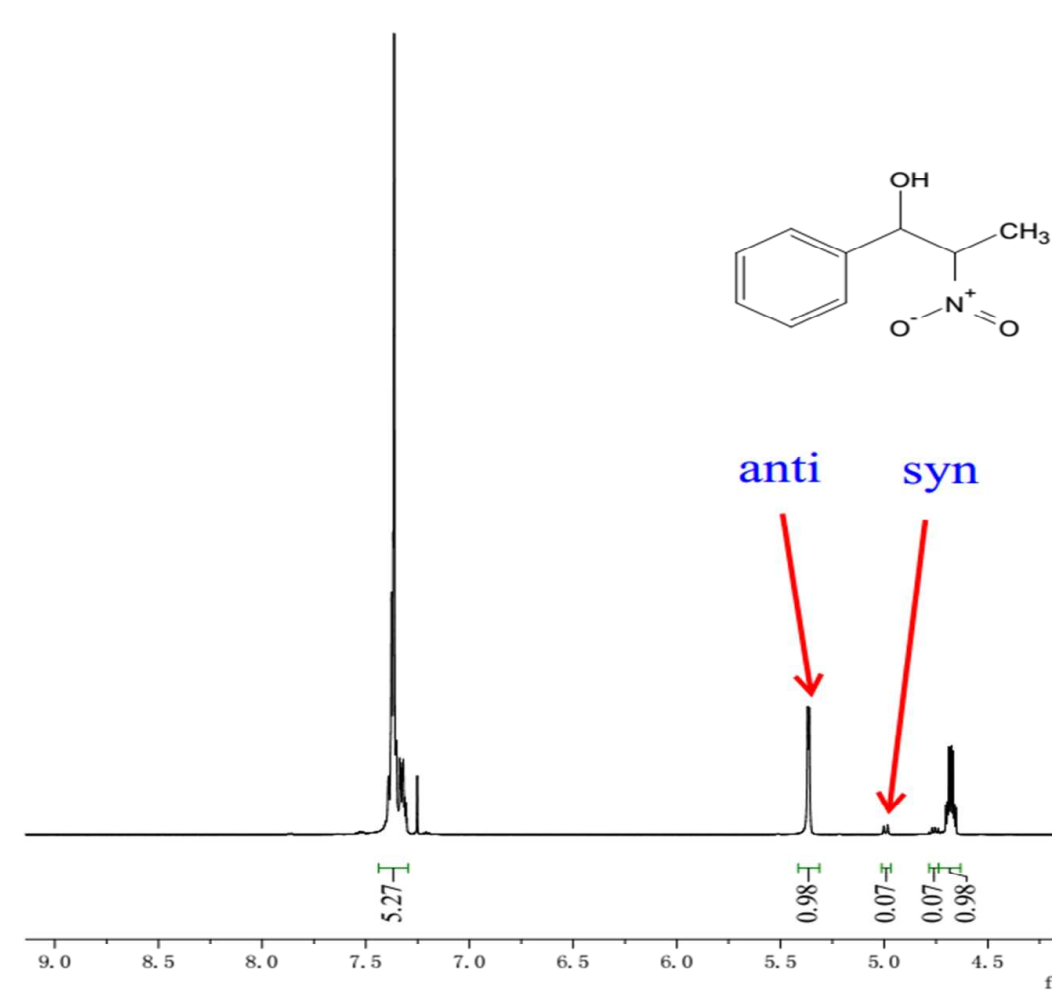

anti syn
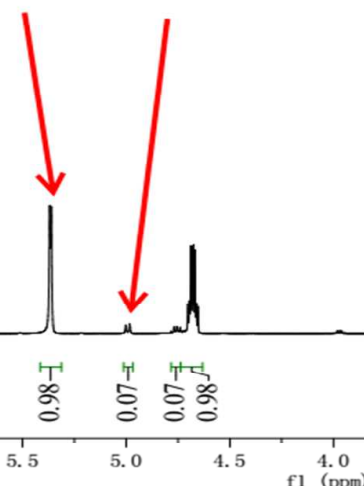

(ppm) 
4ba (Entry 2, Table2)
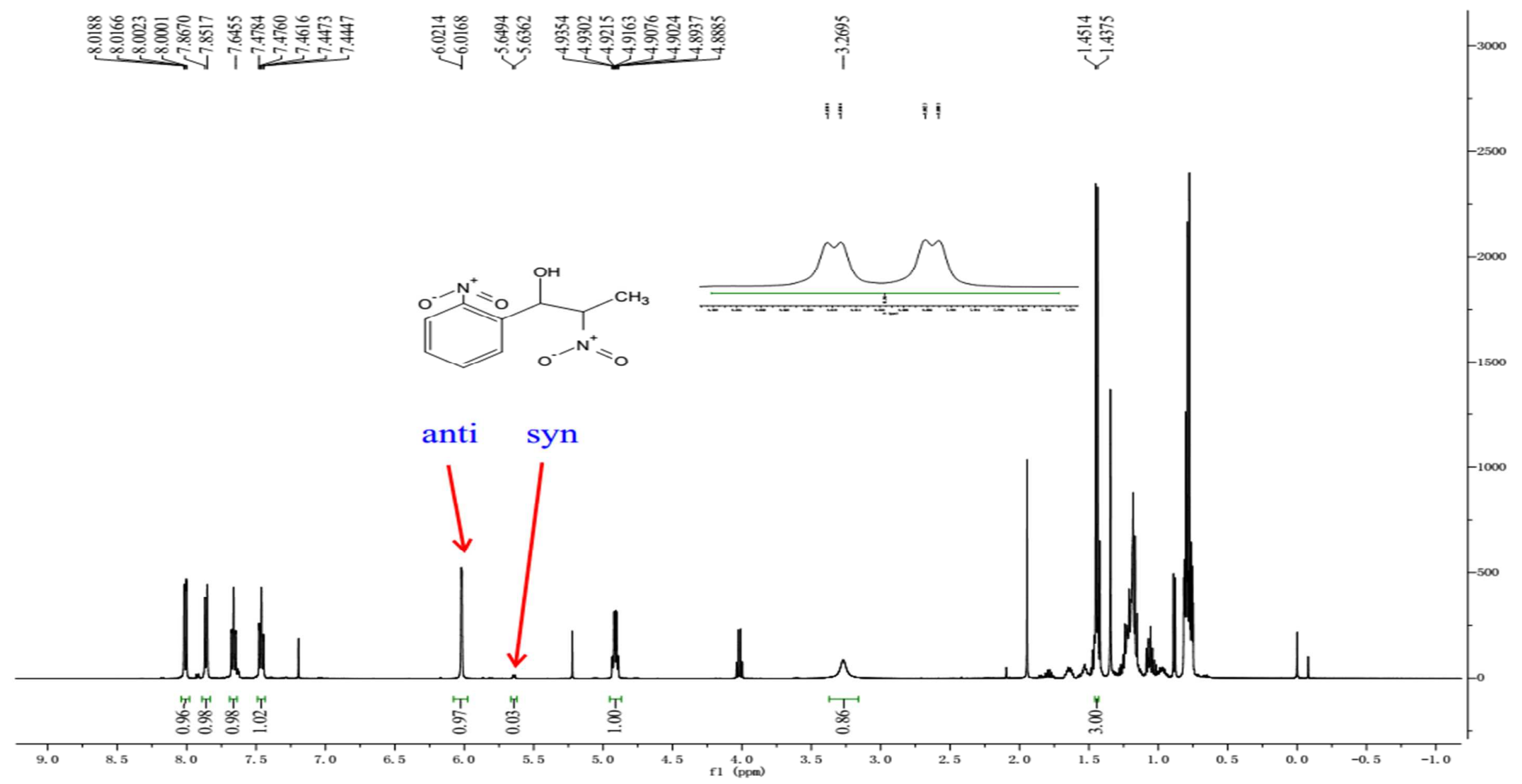
4ca (Entry 3, Table 2)

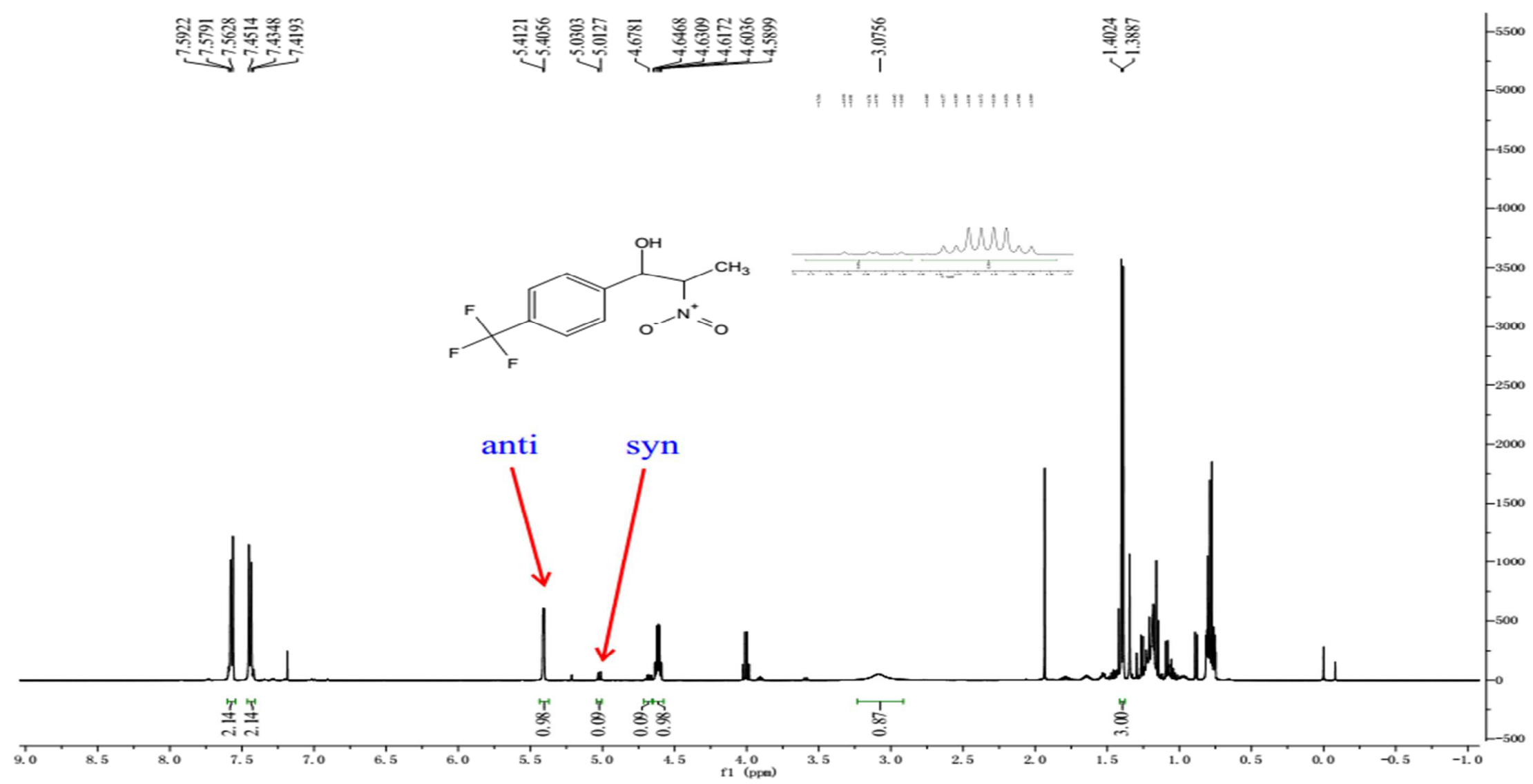


4da (Entry 4, Table 2)

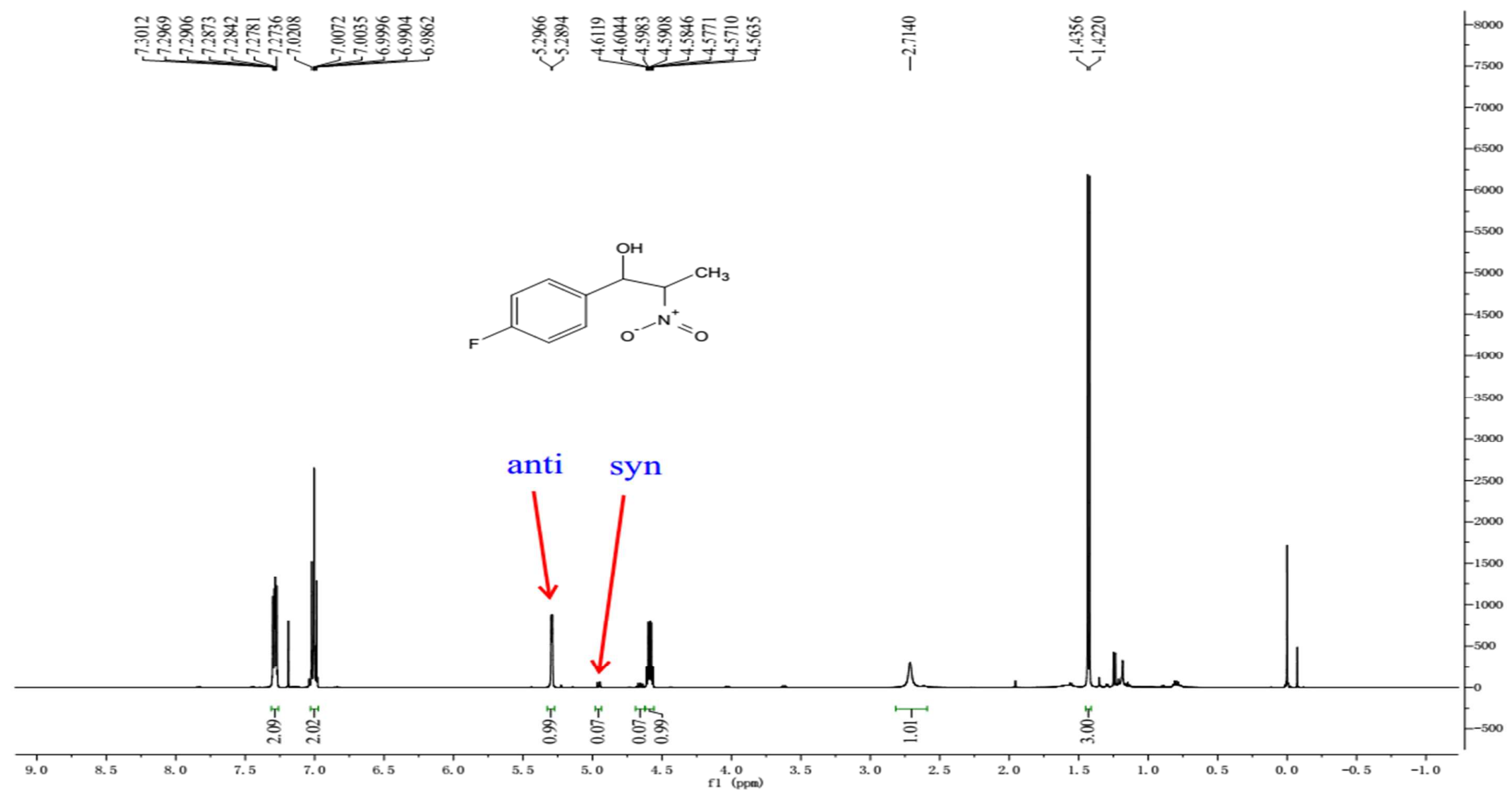


4ea (Entry 5, Table 2)

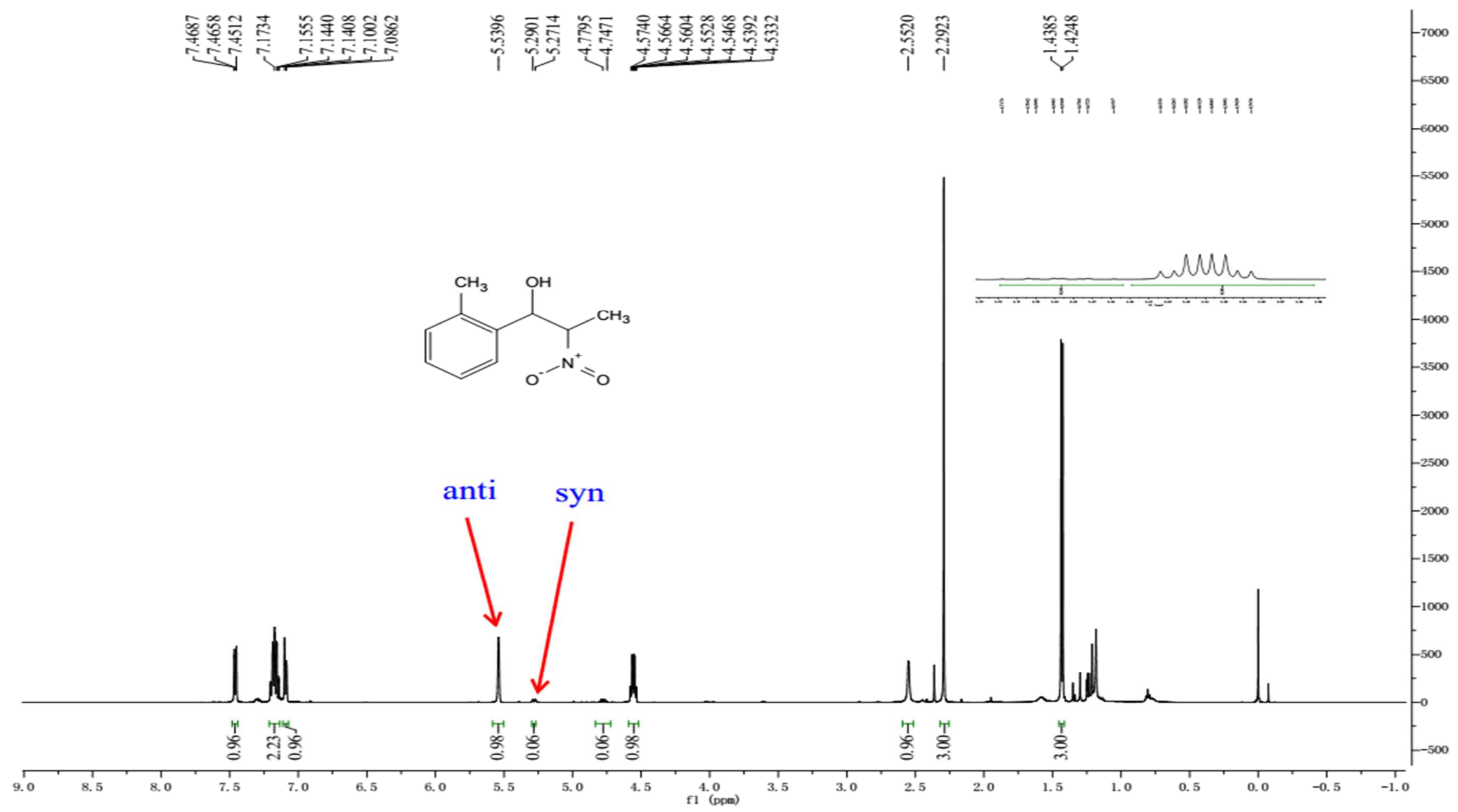


4fa (Entry 6, Table 2)

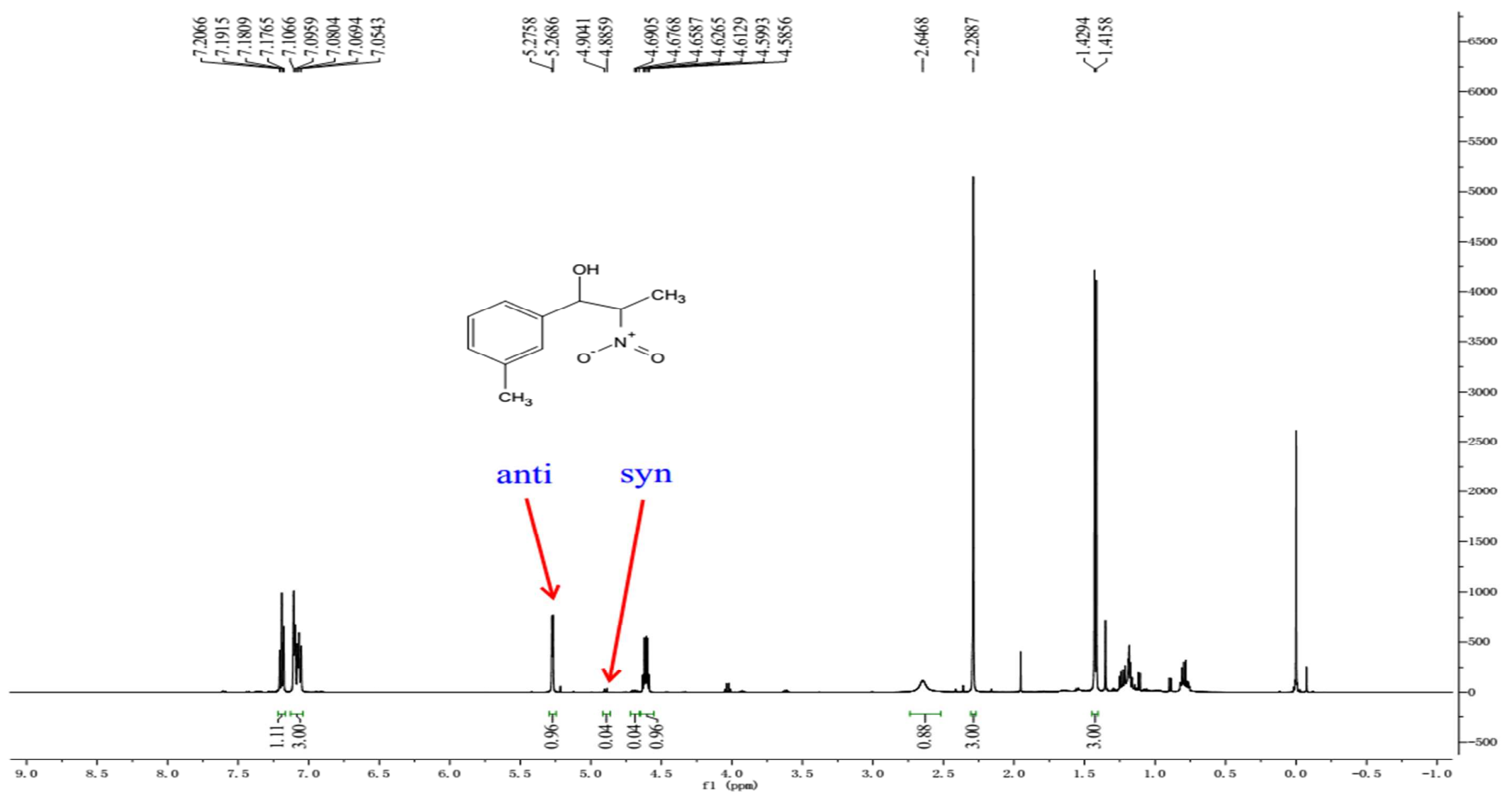


4ga (Entry 7, Table 2)

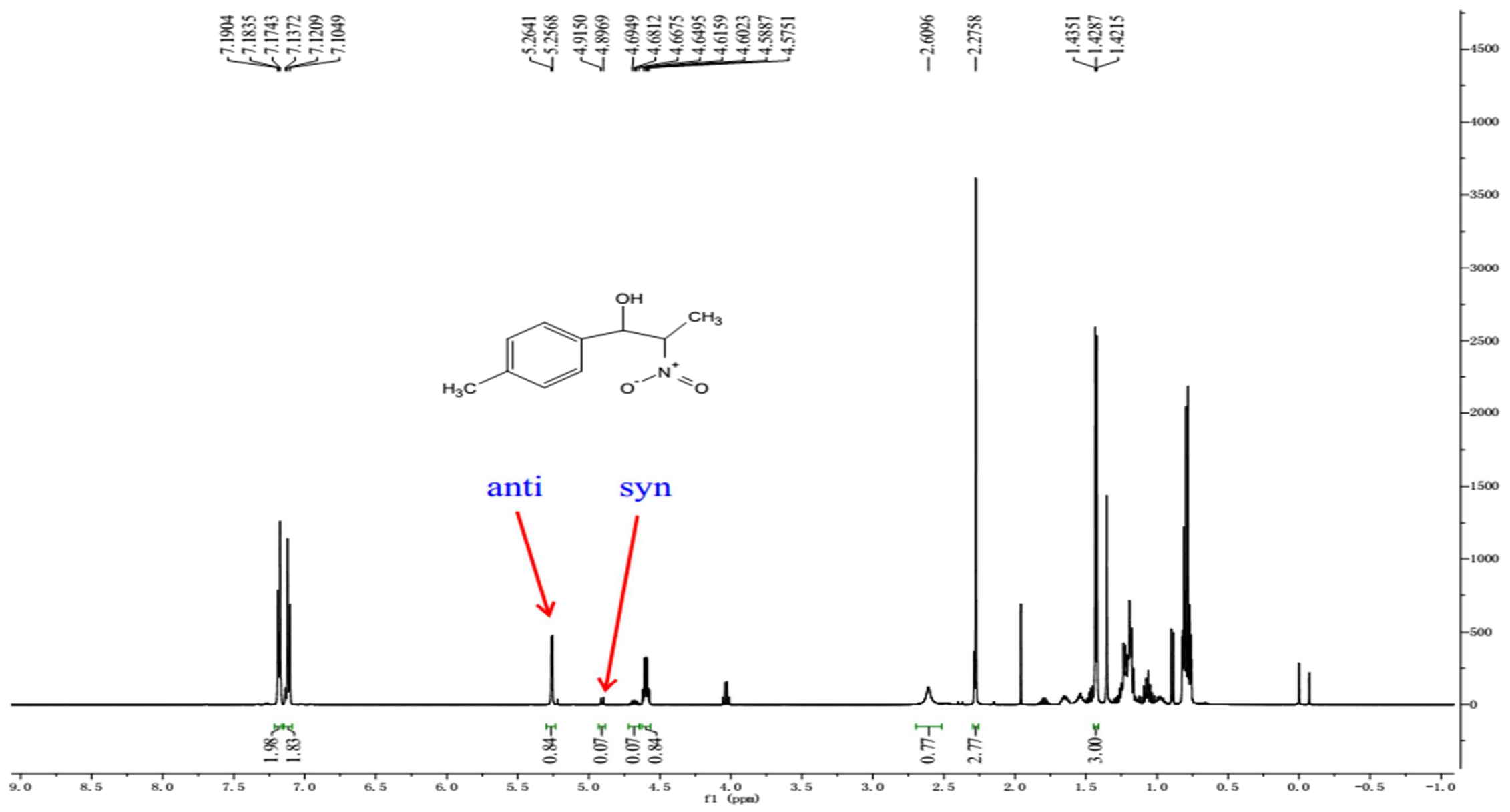


4ha (Entry 8, Table 2)

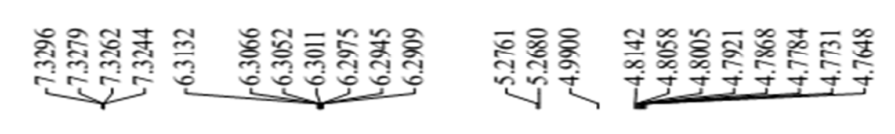

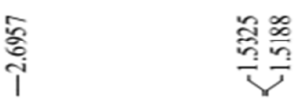

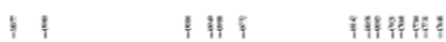
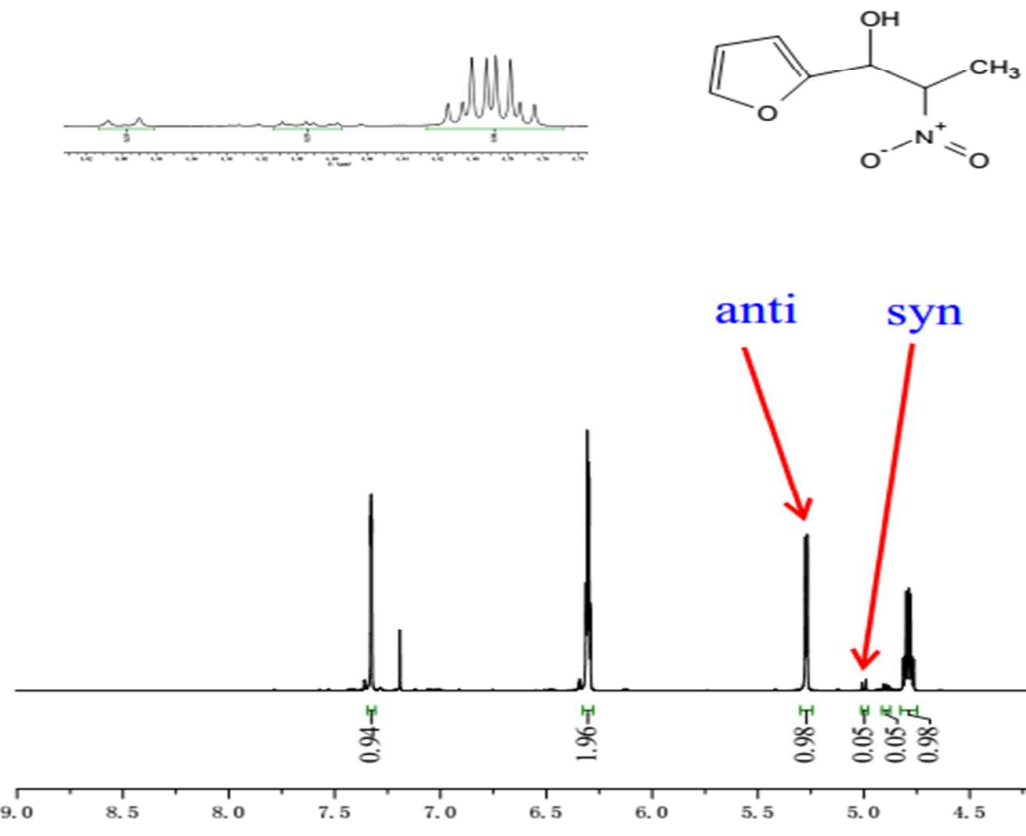

syn

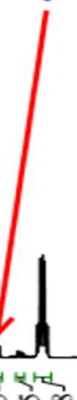

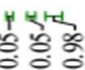

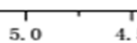


4ia (Entry 9, Table 2)

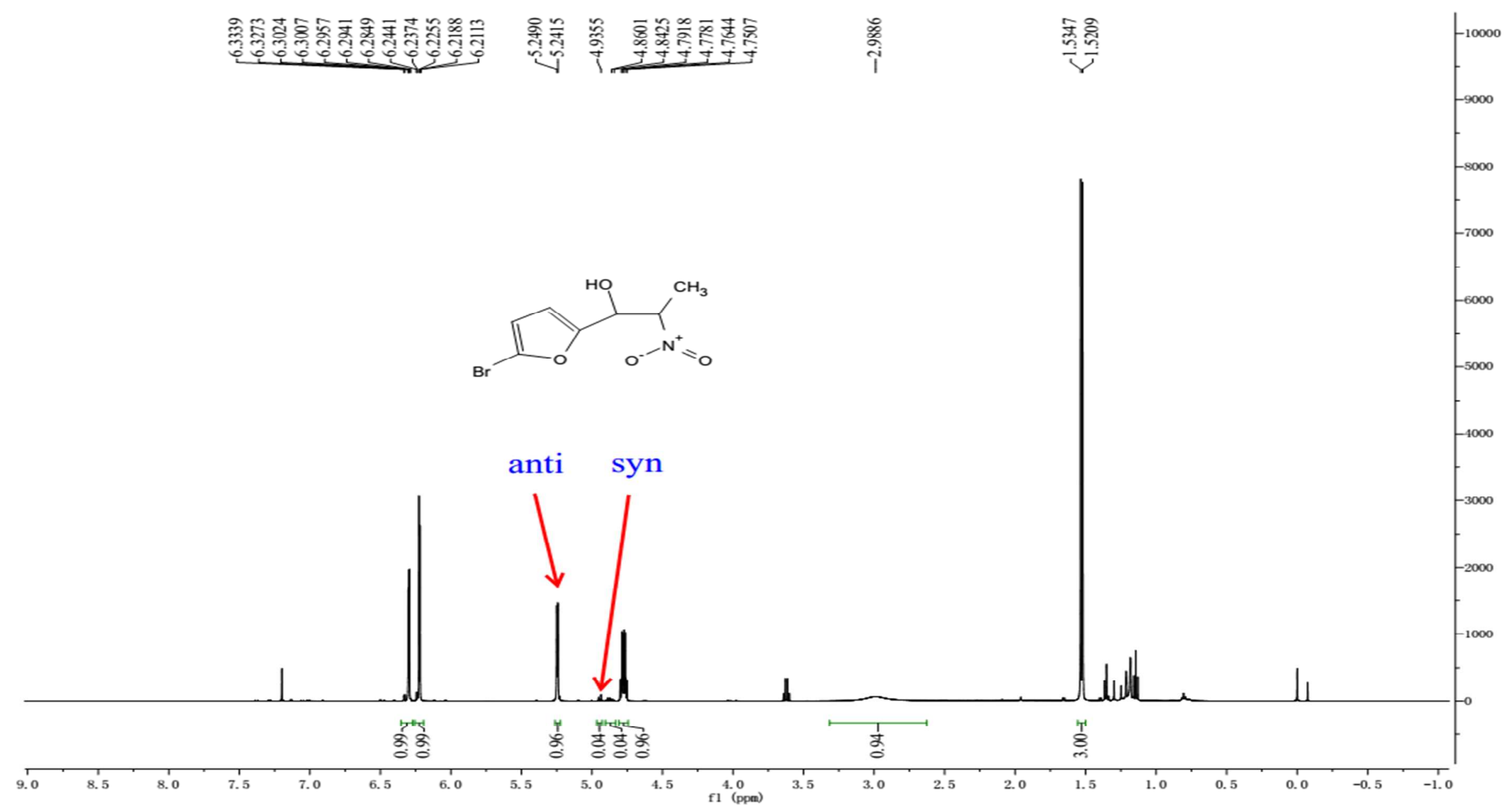


4ia (Entry 9, Table 2)

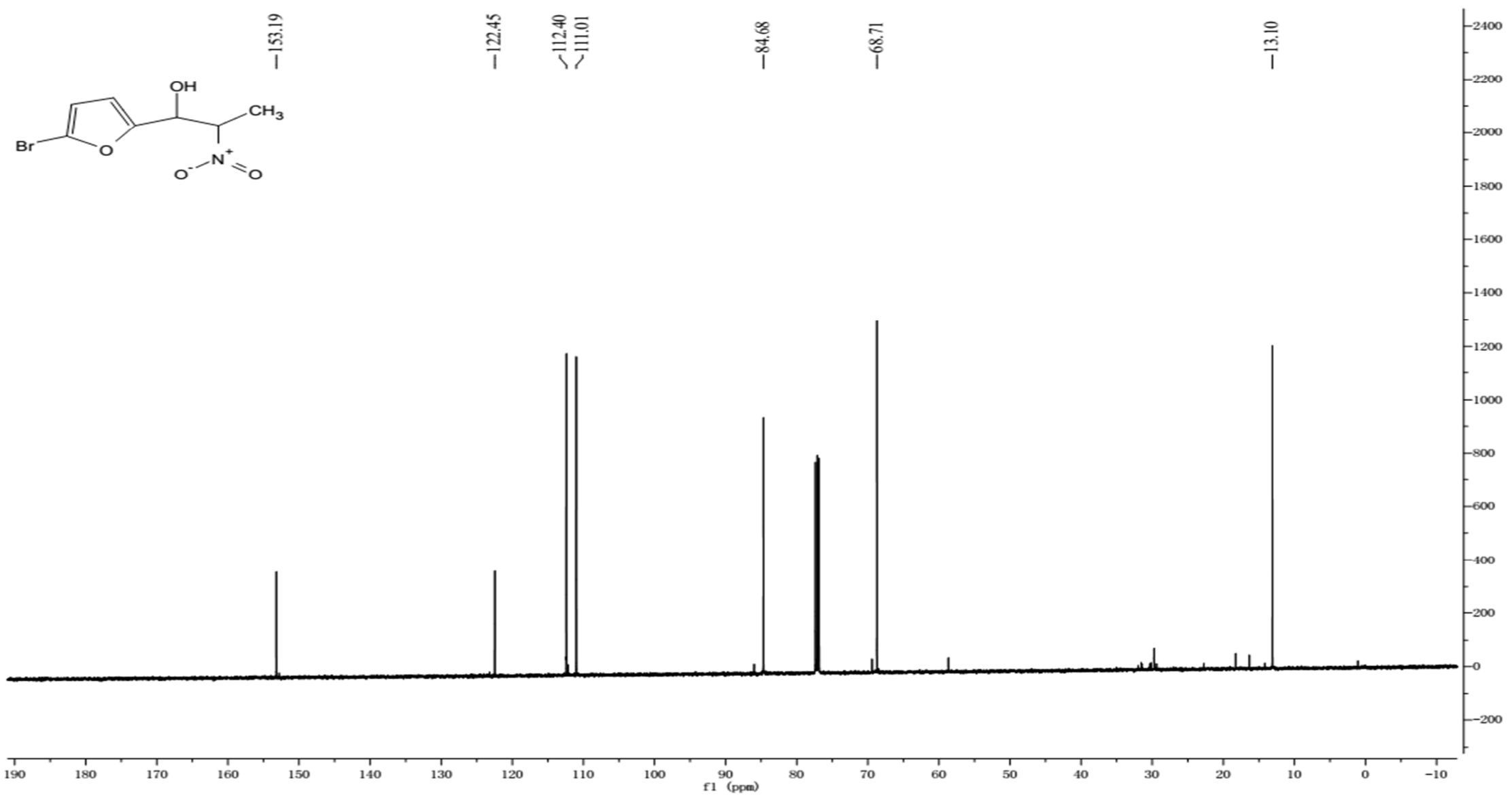


4ja (Entry 10, Table 2)

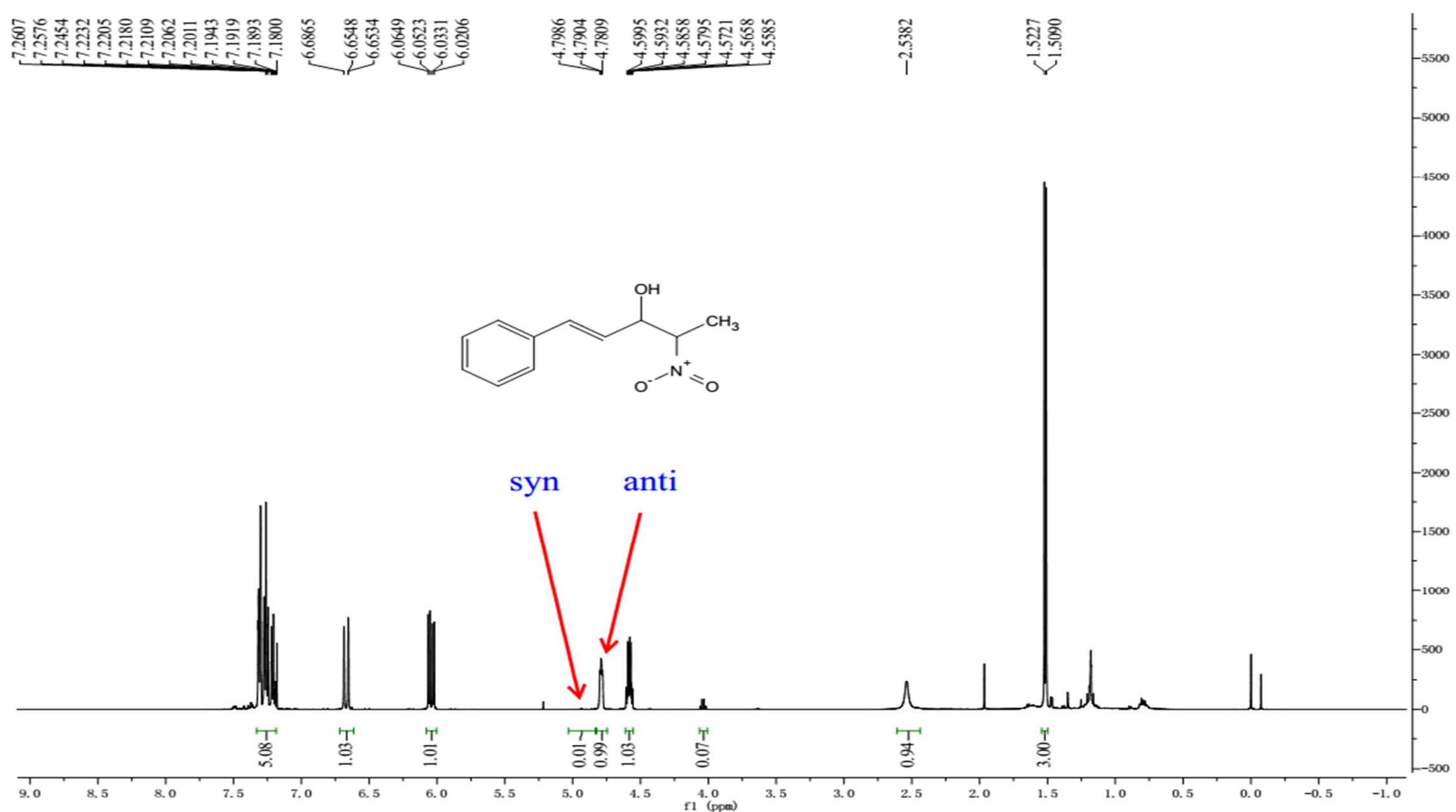


4 ka (Entry 11, Table 2)

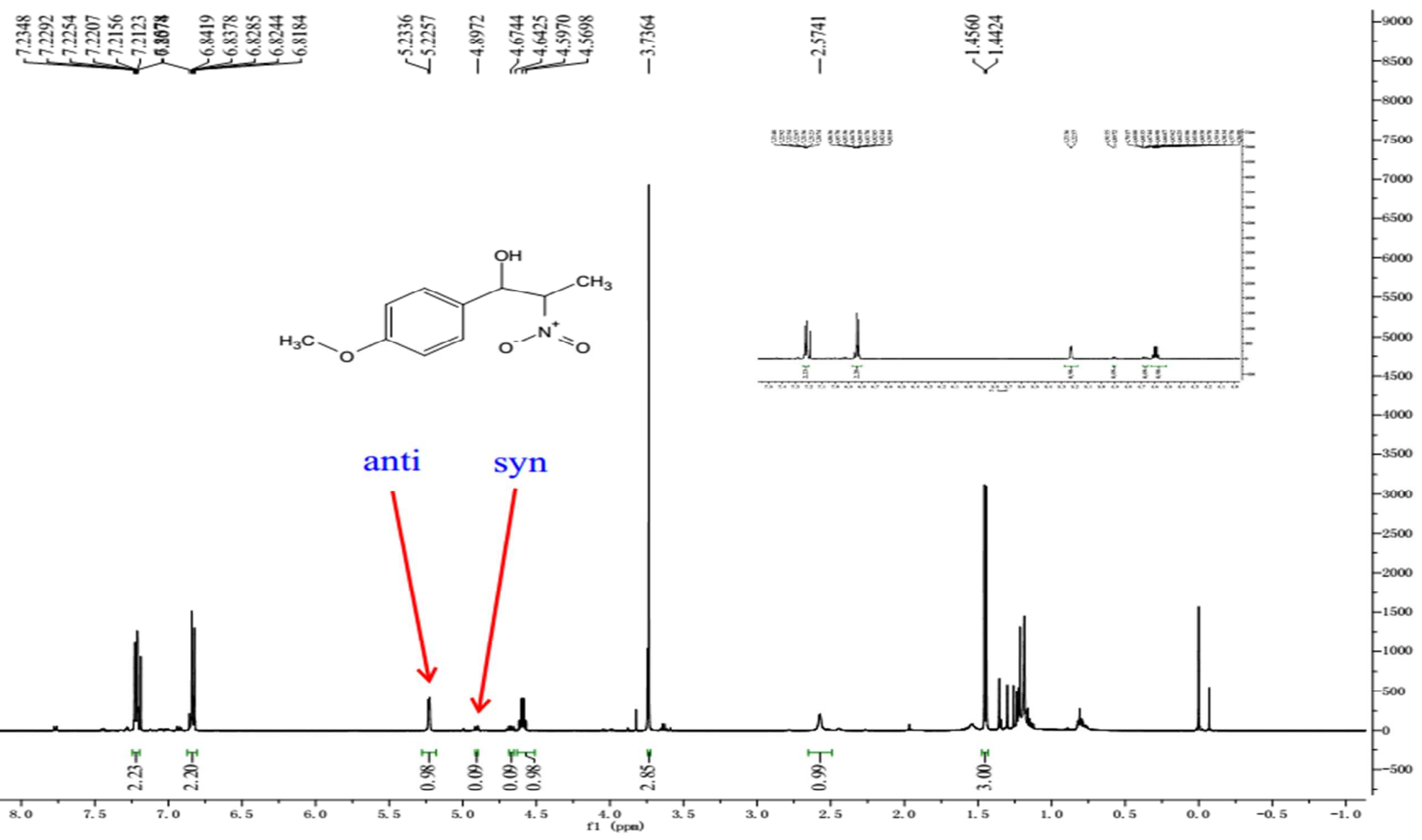


4la (Entry 12, Table 2)

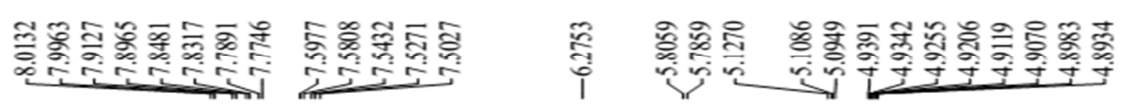

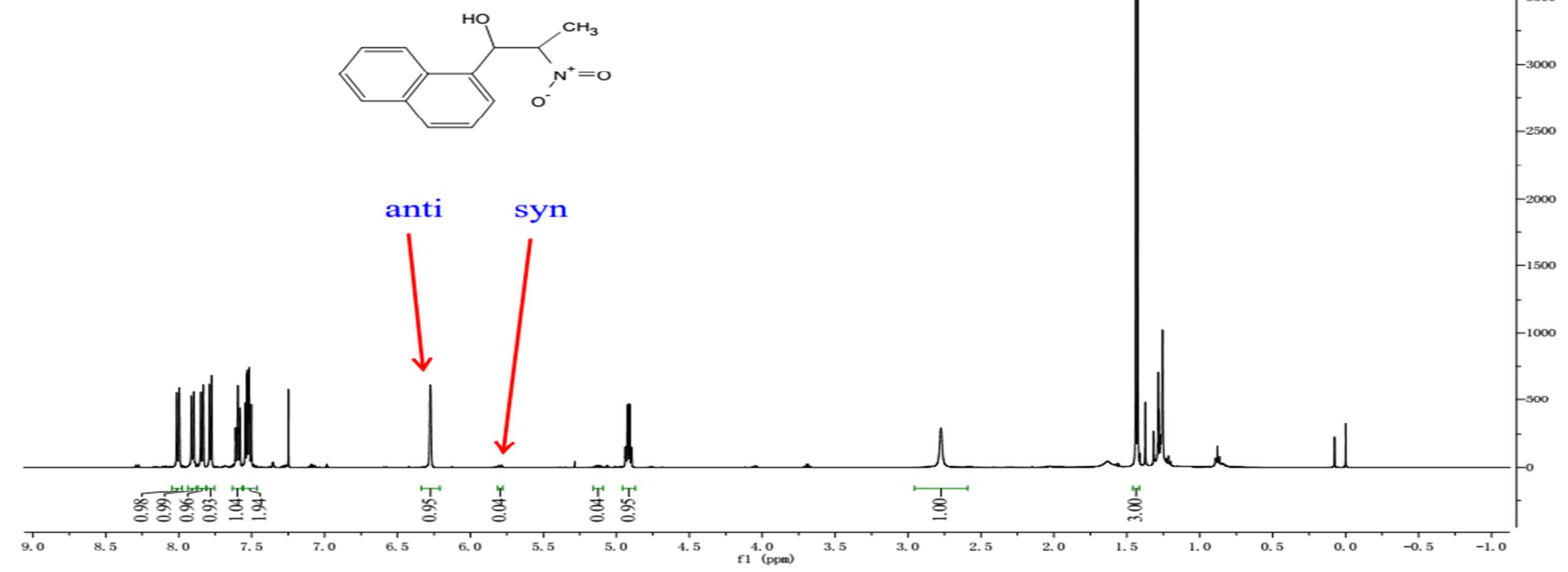


4ma (Entry 13, Table 2)

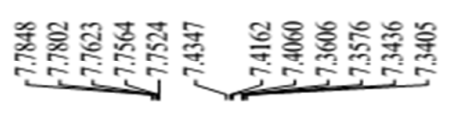

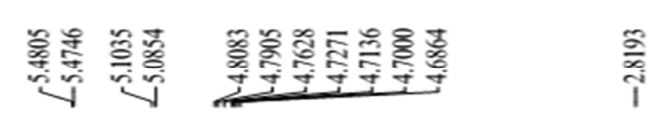

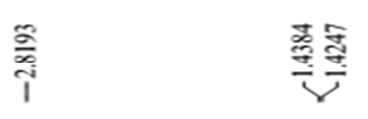

$-4000$

414374959191
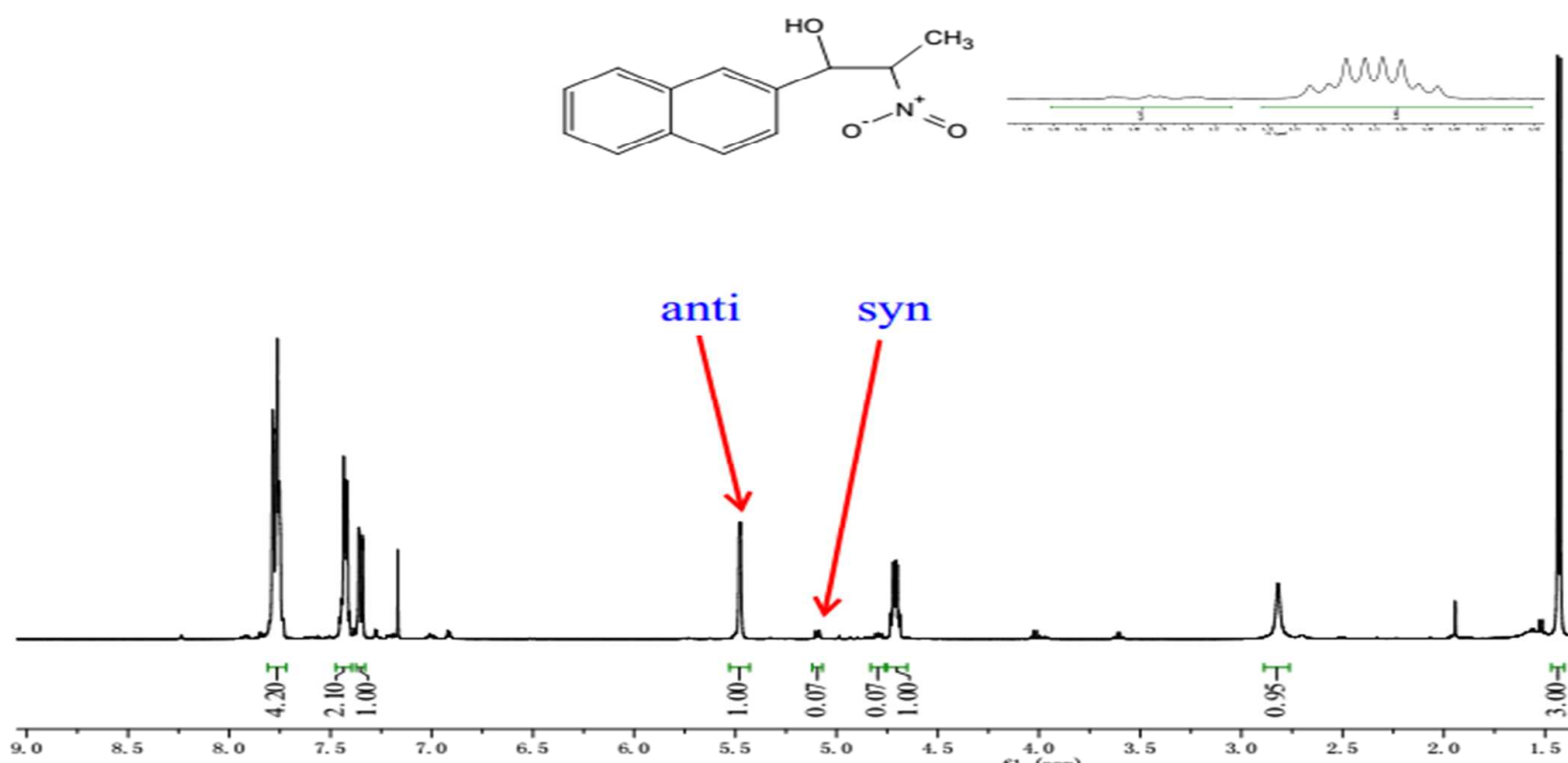

anti syn
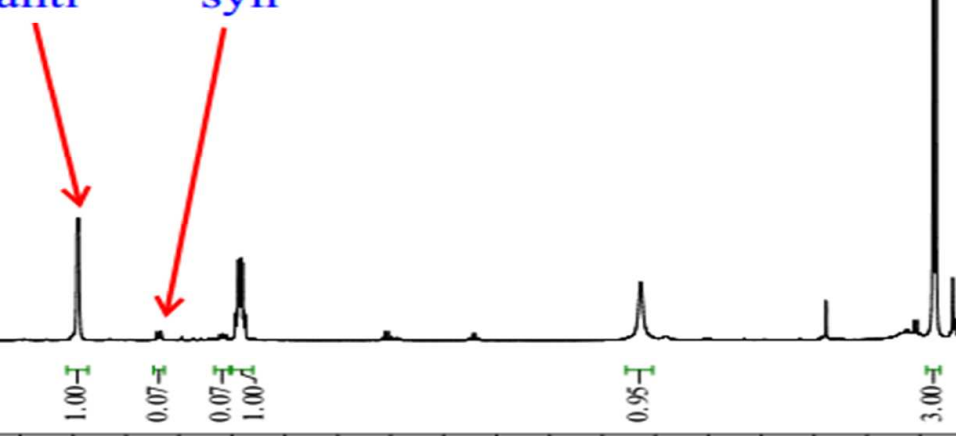
4na (Entry 14, Table 2)

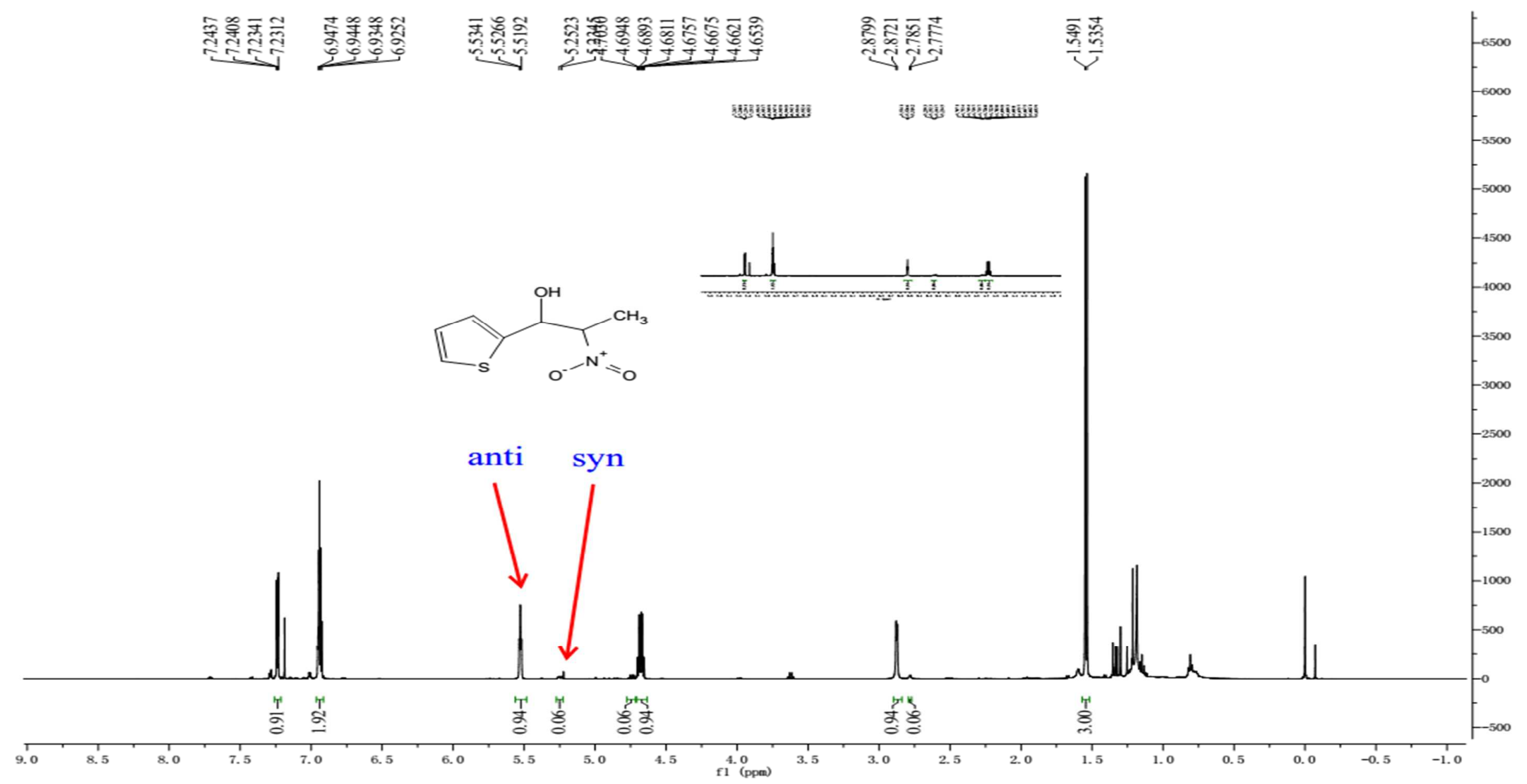


4oa (Entry 15, Table 2)

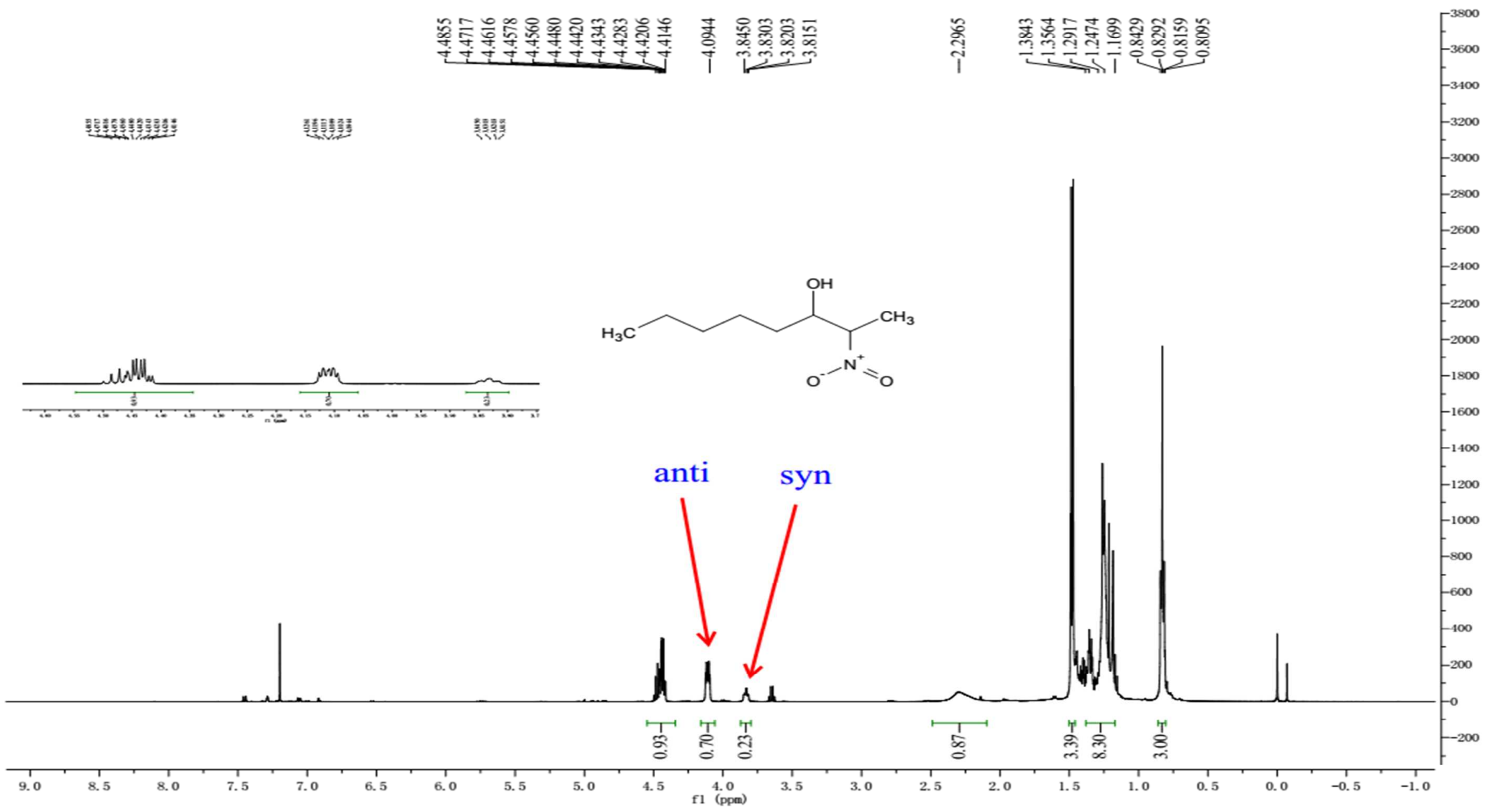


4ab (Entry 16, Table 2)
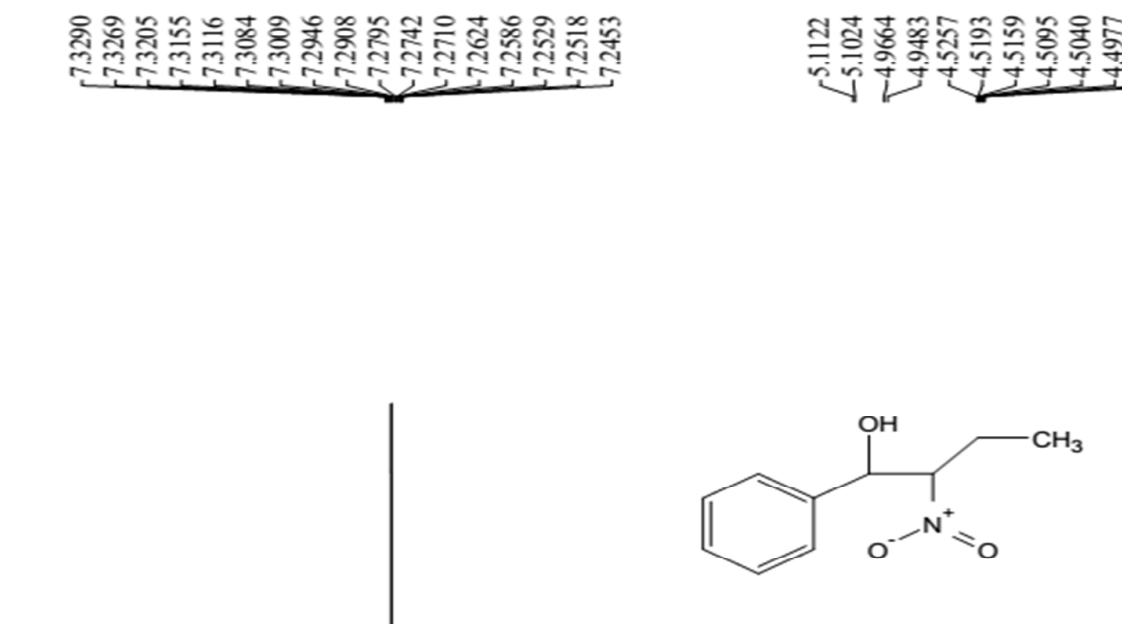

anti syn
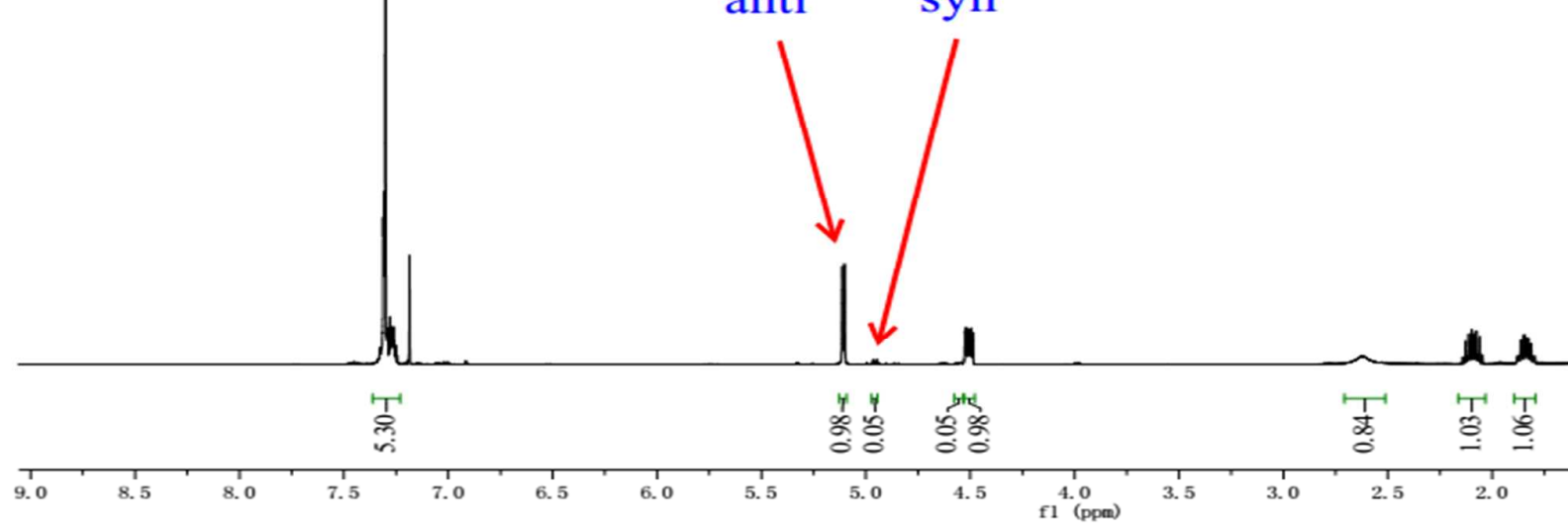